\title{
Applying Thermomechanical Analogy to Predict the Arterial Residual Stress State
}

\author{
Janez Urevc1,* - Milan Brumen², 3 - Vojko Flis ${ }^{4}$ - Boris Štok ${ }^{1}$ \\ 1University of Ljubljana, Faculty of Mechanical Engineering, Slovenia \\ 2University of Maribor, Faculty of Medicine, Slovenia \\ 3 Jožef Stefan Institute, Slovenia \\ ${ }^{4}$ University Medical Centre Maribor, Department of Vascular Surgery, Slovenia
}

The prediction of the arterial zero-stress state is as of yet an unresolved problem in the field of modelling the mechanical response of patientspecific arteries. This is because the configuration associated with arterial zero-stress state is impossible to obtain experimentally. However, the zero-stress configuration (or, equivalently, the residual stresses related to this configuration) represents the crucial data of any numerical analysis.

In this study, the mechanical response of a pre-stressed, pressurised, hyperelastic tube (representing the artery) is determined without knowing the initial zero-stress configuration of the artery. Instead, to predict the arterial residual (bending and stretching) stresses, a corresponding thermomechanical analogy is used. As shown in the paper, the arterial residual stress state can equally be obtained by thermally loading a properly defined closed tube. Thus, based on the loaded state of the corresponding thermomechanical model, the arterial residual stress sate is constructed, from where the arterial loaded state can be obtained. The initial configuration of the thermomechanical model is defined on the basis of the arterial loaded configuration. The methodology is validated by predicting the zero-stress state of the artery. The predicted equilibrium state of the artery, when cut longitudinally and transversally, has the form of an opened-up tube with a relatively low stress state in comparison to the arterial residual stresses. The results thus demonstrate that arterial residual stresses that are predicted with the corresponding thermomechanical model exhibited the bending distribution, which proves the methodology to be adequate. Keywords: residual stresses, zero-stress state of arteries, finite element method, thermomechanics

Highlights

- $\quad$ Predicting the arterial mechanical response based on the in vivo arterial data.

- $\quad$ Proposed methodology for predicting the arterial mechanical response.

- $\quad$ Using thermomechanical analogy for predicting the arterial residual stress state.

- $\quad$ Predicting the arterial zero-stress state based on the in vivo arterial data.

- Determination of bending stresses by means of thermomechanical analogy.

\section{O INTRODUCTION}

The progress of medical imaging has enabled the increasingly accurate acquisition of patient-specific arterial geometries. This, in turn, allows for the study of arterial response under various conditions, giving additional insight into human physiology that cannot be experimentally obtained. Medical imaging is most often performed in vivo, thus providing the arterial geometry that corresponds to the arterial loaded (i.e., physiological) state. To numerically model the arterial mechanical response, however, either the arterial zero-stress configuration or the arterial residual stress state is needed. This study addresses the problem of modelling the arterial response in cases when the arterial zero-stress configuration is not given whereas the arterial loaded configuration is known. The artery is treated as a hyperelastic, residually pre-stressed and pressurised tube.

The arterial unloaded state represents a part of the artery removed from the surrounding tissue, freed of intraluminal pressure, and removed from the body. In this state, the artery is free of external loads but is still subject to some amount of stresses, referred to as the residual stresses. These stresses are relieved by cutting the artery longitudinally. The artery then springs open and assumes a sector-like shape in which the size of this opening quantifies the magnitude of the relieved residual stresses. Because of their transmural distribution, with compressive stresses on the arterial inside and tensile stresses on the outside [1], the residual stresses are normally associated with pure bending stresses [2]. Although some residual stresses exist in the arterial longitudinal direction [3] and [4], we will assume that only one longitudinal cut is sufficient to release almost all residual stresses [5] and [6]. In this work, the artery is treated as single-layered structure. Even though this is a rough approximation of the complex structure of an artery, it is able to provide a reasonable assessment of the arterial physiological response [7]. 
Residual stress produces a favourable (physiological) environment for cell functions [8], which is created by lowering the gradient of the transmural stresses [9] and [10]. According to the so-called uniform strain hypothesis [11], the distribution and magnitude of the residual stresses cause the physiological intraluminal distribution of the circumferential strain $\lambda_{\phi}(r)$ to be uniform $\left(\lambda_{\phi}(r)=\right.$ const $)$, which implies a low gradient of transmural stresses. Furthermore, it has been experimentally observed that the distribution of the residual stresses in the vessel wall is three dimensional, meaning that they depend on both the radial position and on the axial position [5], [12] and [13].

A crucial datum needed for any numerical prediction of the arterial response, as already mentioned, is the zero-stress configuration of the artery. To the knowledge of the authors, the only methodology that allows the inclusion of residual stresses in a patient-specific arterial geometry is presented in the study performed by Alastrue et al. [2]. There, the residual stresses are accounted for by applying a simplified initial strain field that is obtained from a computation of the residual stress field in a circular arterial segment. The drawback of this approach is that the zero-stress configuration of the circular segment, which must be adopted from the literature, does not correspond with a patientspecific artery. Furthermore, due to the complexity of the problem, various simplifications have been made in the literature in order to obtain the patientspecific arterial zero-stress configuration. The most general approach is to take the loaded configuration of the artery for the arterial initial stress-free state. This approach leads to unphysically large arterial deformations [14] where, subsequently, the predicted arterial configuration obtained by pressurising the artery with intraluminal pressure strongly differs from the one observed in vivo. Another approach is to neglect the arterial residual stresses and assume the arterial unloaded configuration for the arterial initial zero-stress configuration. For example, in the study conducted by Gee et al. [14], two such methods are presented that enable a prediction of the pre-stress state of a patient-specific geometries under in vivo loads. The first is the inverse design (ID) analysis. In this approach, which is also used in [15] and [16], the zero-stress configuration is computed and then used to compute a (non-unique) strain and stress state that satisfied equilibrium with respect to the known spatial configuration. The second method is based on a modified updated Lagrangian formulation (MULF), in which a multiplicative split of the deformation gradient is used to produce a displacement-free prestrained/pre-stressed state. Despite the widespread use of the first method in particular, the drawback of such an approach is that it neglects the residual stresses. On the other hand, progress has been made in obtaining the arterial unloaded state starting from the opened-up configuration (see for instance [12], [17] and [18]). In this case, however, the arterial opened-up configuration needed to be provided (i.e., given).

In this paper, a new methodology is presented that predicts the mechanical response of a hyperelastic, residually pre-stressed and pressurised tube (representing an artery). The loaded configuration of the artery is assumed known whereas the arterial opened-up zero-stress configuration is unknown. The methodology for predicting the arterial residual (i.e., bending and stretching) stresses is based on an equivalent approach where a corresponding thermomechanical problem of an associated closed tube is defined. In Section 1, a case study is presented and the assumptions of the considered mechanical problem are specified. Section 2 gives a theoretical background on (i) the characterisation of the circular arterial segment, (ii) the thermomechanical analogy for obtaining the arterial residual stresses, and (iii) the use of the thermomechanical analogy to predict the mechanical response of a realistic arterial segment. The methodology presented for the arterial segment is then generalised to treat a real artery in Section 3. The analysis results obtained by applying the developed methodology to the case study are given in Section 4 together with a corresponding validation of the methodology. Finally, in Section 5, the conclusions are given along with some guidelines for future research.

\section{PROBLEM DEFINITION}

The methodology for predicting the arterial residual stress state is demonstrated using a case study analysis considering an analytically defined, hyperelastic tube. The tube, here referred to as the arterial model (AM), is defined so as to resemble the in vivo observed artery. The inner, i.e., the luminal surface of the AM $\left(\Gamma_{i}^{\mathrm{AM}}\right.$, Fig. 1a) is defined as a straight tube of an elliptical cross section having respective principal directions coincident with the $x$ and $y$ axis along the entire AM. The assumed variation of the elliptical semi-minor and semi-major axis $(a(z)$ and $b(z))$, specifying the surface $\Gamma_{i}^{\mathrm{AM}}$, is shown in Fig. $1 \mathrm{~b}$. Based on this variation, the AM luminal crosssectional area $A(z)$ variation is computed as show in Fig. 1b. Furthermore, the thickness $t$ of the AM is assumed to be constant along the whole section, its 


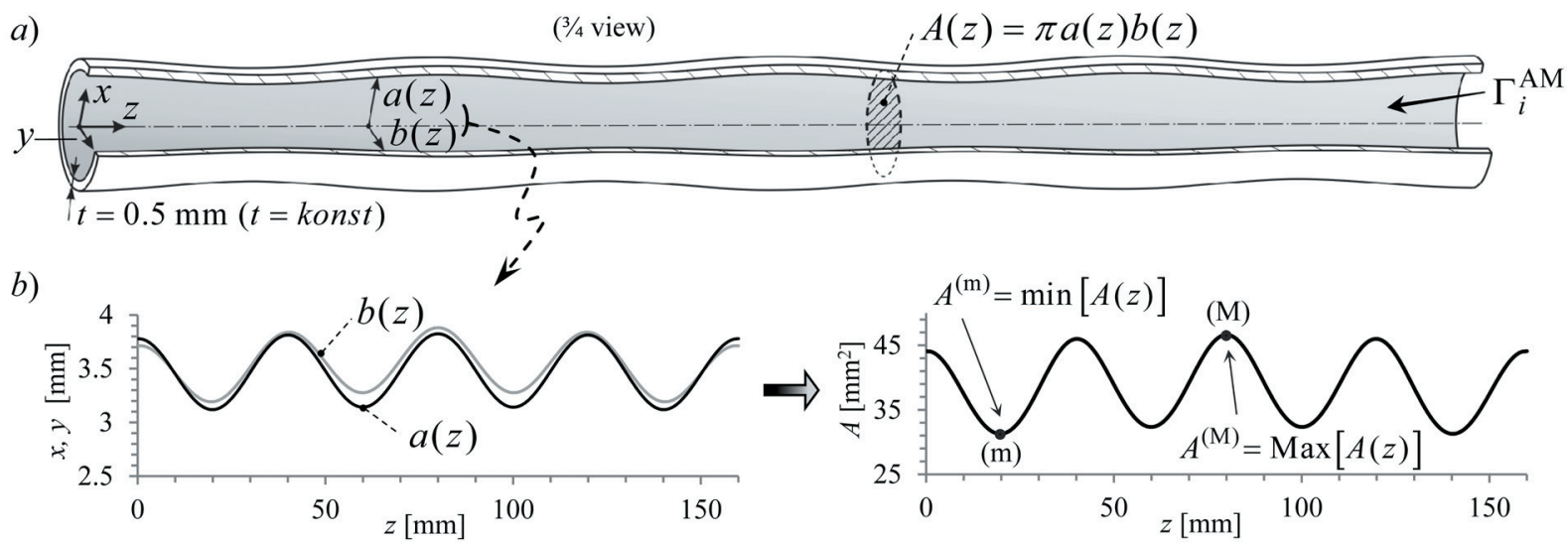

Fig. 1. Presentation of the case-study: a) Three-dimensional representation of the arterial model (AM); b) the inner, i.e., the luminal surface of the $\mathrm{AM}\left(\Gamma_{i}^{\mathrm{AM}}\right)$ represents a straight tube of an elliptical cross section which is defined by the ellipse semi-axes a(z) and b(z)

value being $t=0.5 \mathrm{~mm}$. The length of the model is $160 \mathrm{~mm}$.

Let us assume that the AM geometry is obtained from medical imaging and that the configuration presented in Fig. 1a corresponds to the AM diastolic state with an intraluminal pressure of $P=70 \mathrm{mmHg}$. For the material behaviour of the AM, an isotropic hyperelastic material model is assumed, described by a strain energy density function $W[17]$ :

$$
W=\frac{c_{a}}{c_{b}}\left[\exp \left[\frac{c_{b}}{2}\left(I_{1}-3\right)\right]-1\right],
$$

where $I_{1}$ is the first invariant of the strain tensor and $c_{a}$ and $c_{b}$ are the constitutive parameters. In [17], their values were experimentally determined to be $c_{a}=44.2 \mathrm{kPa}$ and $c_{b}=16.7$.

Remark: The artery is treated as a singlelayered tube with isotropic properties. This is an oversimplification because it is well known that arteries are composed of three distinct layers (intima, media and adventitia), which have, in general, anisotropic properties [19]. Nevertheless, as discussed in the introduction, the considered AM is still able to provide a reasonable approximation of the arterial response. In this regard, when taking into consideration that the primary goal of this paper is to give a presentation of the new developed methodology, the adopted model approximation may be considered acceptable and justified for the given purpose. Additionally, the effect of the tissue surrounding the artery, which acts as a pressure load on the arterial outer surface, is neglected in this study.

\subsection{Arterial Configurations}

The arterial configurations are denoted by the symbol $\Omega$. The subscript supplementing $\Omega$ specifies the corresponding arterial loading state whereas the superscript of $\Omega$ indicates the corresponding model. The superscript "AM" will refer to the arterial model and " $C$ " to the configurations of the circular arterial segments. The following arterial configurations are considered (Fig. 2):

Loaded state $\left(\Omega_{2}\right)$ : In its loaded state the artery is subject to intraluminal pressure $P$ and longitudinal (axial) as well as circumferential pre-stretch.

Stretched state $\left(\Omega_{1 \lambda}\right)$ : The arterial stretched state is obtained by removing the pressure $P$ from $\Omega_{2}$. (Note that the term arterial stretched state is not common in the literature; it is defined here because it offers a good targeting state for the methodology presented in the following sections.)

Unloaded state $\left(\Omega_{1}\right)$ : The unloaded state is obtained by releasing the axial pre-stretch from $\Omega_{1 \lambda}$. The associated axial contraction is described by the axial stretch $\lambda$. Since the in vivo data is insufficient to determine $\lambda$ [20], we have adopted here the data of Delfino et al. [17], where $\lambda$ is set to $10 \%(\lambda=1.1)$.

Zero-stress state $\left(\Omega_{0}\right)$ : Once the artery in its unloaded state is cut open, it is assumed that the stresses in the artery are close to zero. The obtained state is taken as the arterial zero-stress state. The axial contraction $\Lambda$, that is associated with the longitudinal cut is normally neglected, therefore $\Lambda=1$ [17]. 


\section{MECHANICAL RESPONSE OF A CIRCULAR ARTERIAL SEGMENT}

The subsequent subsections begin with some theoretical background on the characterisation of the circular arterial segment, and are followed by a presentation on an analogical approach aimed to obtain the stretched state of a circular arterial segment. The latter is achieved by defining a corresponding thermomechanical problem of a closed circular tube. Finally, the application of this analogy to predict the stretched state of a realistic arterial segment is schematically presented.

\subsection{Stress Analysis of a Circular Arterial Segment}

The mechanical response of an arterial segment (i.e., a slice of the artery) can be characterised by simplifying the segment geometry to a straight circular tube [20] to [23]. The zero-stress state configuration of the open circular segment $\Omega_{0}^{\mathrm{C}}$ (Fig. 2) is thus defined with the radii $R_{i}$ and $R_{o}$ and the opening angle $\Theta_{0}$. The unloaded state configuration $\Omega_{1}^{\mathrm{C}}$ is then obtained by bending (closing) the segment from its zero-stress state configuration $\Omega_{0}^{\mathrm{C}}$. By further applying the axial stretch $\lambda$ and the inner pressure $P$ to the unloaded state configuration $\Omega_{1}^{\mathrm{C}}$, the stretched state configuration $\Omega_{1 \lambda}^{\mathrm{C}}$ and the loaded state configuration $\Omega_{2}^{\mathrm{C}}$ of the segment are obtained, respectively.

The deformation gradient tensor $\mathbf{F}$ associated with the loaded state configuration $\Omega_{2}^{C}$ accounts for all three successive motions of material particles, displayed in Fig. 2. A material particle $\left(r_{i} \leq r \leq r_{o}\right)$ can be represented in terms of the principal stretches as:

$$
\mathbf{F}=\operatorname{diag}\left[\frac{\mathrm{d} r}{\mathrm{~d} R}, \frac{\pi r}{\Theta_{0} R}, \lambda \Lambda\right]=\operatorname{diag}\left[\lambda_{r}, \lambda_{\phi}, \lambda_{z}\right],
$$

where $\lambda_{r}, \lambda_{\phi}, \lambda_{z}$ are, respectively, the principal stretches in the radial, circumferential, and axial directions. The right Cauchy-Green tensor is defined as $\mathbf{C}=\mathbf{F}^{\mathrm{T}} \mathbf{F}$, where $\mathbf{F}^{\mathrm{T}}$ is the transpose of $\mathbf{F}$. The reference radius $R(r)$ of the material particle in the zero-stress state configuration $\Omega_{0}^{C}$ is determined by imposing the incompressibility condition $J=\operatorname{det} \mathbf{F}=\lambda_{r} \lambda_{\phi} \lambda_{z}=1$ and considering the functional expressions for the principal stretches given in Eq. (2):

$$
R=\left(R_{o}^{2}-\frac{\pi \lambda \Lambda}{\Theta_{0}}\left(r_{o}^{2}-r^{2}\right)\right)^{1 / 2}
$$

In addition, the radial stretch is written as:

$$
\lambda_{r}=1 / \lambda_{\phi} \lambda_{z}=\frac{\Theta_{0} R}{\pi r \lambda \Lambda}
$$

which also comes from the incompressibility condition.

Considering the strain energy density function $W$ the Cauchy stress tensor $\sigma$ is obtained as:

$$
\sigma=-p \mathbf{I}+\bar{\sigma}=-p \mathbf{I}+2 \mathbf{F} \frac{\partial W}{\partial \mathbf{C}} \mathbf{F}^{\mathrm{T}}
$$

where $p$ is the Langrange multiplier, which enforces the incompressibility constraint $\operatorname{det} \mathbf{F}=1$, and $\mathbf{I}$ is the identity tensor. Using Eqs. (1) and (5) the non-zero components of the stress tensor are written as:

$$
\begin{aligned}
& \sigma_{k k}=-p+\bar{\sigma}_{k k}=-p+\lambda_{k}^{2} c_{a} . \\
& \cdot \exp \left[\frac{c_{b}}{2}\left(\lambda_{r}^{2}+\lambda_{\phi}^{2}+\lambda_{z}^{2}-3\right)\right] ; k=r, \phi, z .
\end{aligned}
$$

Because the considered problem is a radial one, all the quantities in the above equation depend only on the radial direction. In the absence of the body forces the equilibrium equations reduce to:

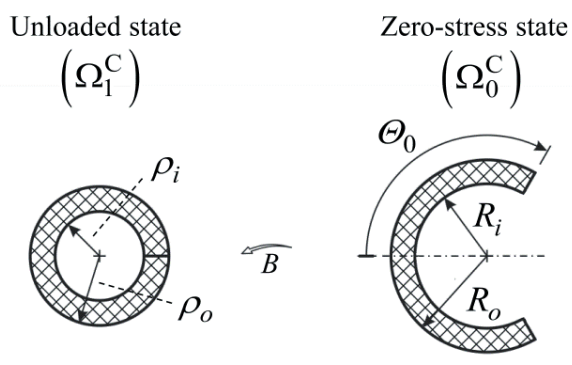

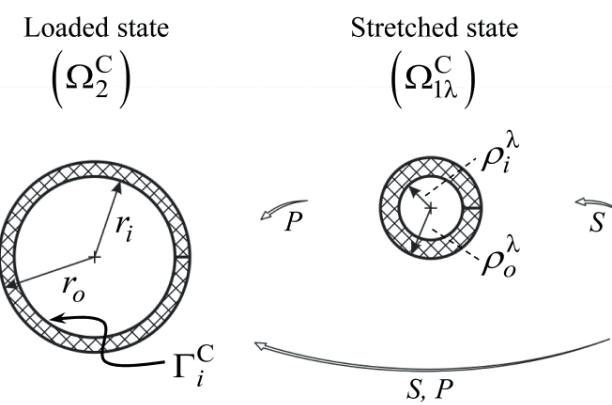

Fig. 2. Configurations of the circular arterial segment: loaded $\Omega_{2}^{\mathrm{C}}$, stretched $\Omega_{1 \lambda}^{\mathrm{C}}$, unloaded $\Omega_{1}^{\mathrm{C}}$ and zero-stress state $\Omega_{0}^{\mathrm{C}}$; the symbols B, S, and P represent bending, axial stretching and pressurising; $\Gamma_{i}^{\mathrm{C}}$ represents the inner (luminal) surface of the loaded state configuration $\Omega_{2}^{\mathrm{C}}$ of the segment 


$$
\frac{\mathrm{d} \sigma_{r r}}{\mathrm{~d} r}+\frac{\sigma_{r r}-\sigma_{\phi \phi}}{r}=0 .
$$

The natural boundary conditions, Eq. (8), are applied both to the inner and outer surface of the segment. The inner surface is subject to the intraluminal pressure $P$, whereas the outer surface is considered traction-free:

$$
\sigma_{r r}\left(r_{i}\right)=-P, \quad \sigma_{r r}\left(r_{o}\right)=0 .
$$

In addition, fulfilment of the equilibrium of the in vivo configuration yields the following expression for the Lagrange multiplier $p$ [24]:

$$
p(r)=P+\bar{\sigma}_{r r}(r)-\int_{r_{i}}^{r}\left(\sigma_{\phi \phi}(r)-\sigma_{r r}(r)\right) \frac{\mathrm{d} r}{r}
$$

and an integral constraint on the stress distribution:

$$
\int_{r_{i}}^{r_{o}}\left(\sigma_{\phi \phi}(r)-\sigma_{r r}(r)\right) \frac{\mathrm{d} r}{r}=P .
$$

By knowing the zero-stress configuration $\Omega_{0}^{\mathrm{C}}$ and the material properties of the segment, the stress state of the segment for a given loaded configuration $\Omega_{2}^{\mathrm{C}}$ is obtained from Eq. (6), after having solved Eq. (9). The stretched state of the segment $\Omega_{1 \lambda}^{\mathrm{C}}$ with the material particles positioned in the interval $\rho_{i}^{\lambda} \leq \rho \leq \rho_{o}^{\lambda}$ (Fig. 2) is obtained from the above equations, if we consider the traction-free boundary condition on both the inner and outer surface of the segment (which means setting $P$ to zero) and the substitution of the integral limits $r_{i}, r, r_{o}$, in Eqs. (9) and (10) with $\rho_{i}^{\lambda}$, $\rho, \rho_{o}^{\lambda}$. In the computation of stresses, the principal stretches computed in accordance with Eqs. (2) to (4) and considering the mapping of $r_{i} \leq r \leq r_{o}$ to $\rho_{i}^{\lambda} \leq \rho \leq \rho_{o}^{\lambda}$ have to be taken into account. The schematic representations of the stresses that occur in the stretched state of the circular arterial segment $\Omega_{1 \lambda}^{\mathrm{C}}$ are shown in Fig. 3.

\subsubsection{Identification of the Zero-Stress State Configuration}

The zero-stress state configuration of the circular arterial segment $\Omega_{0}^{\mathrm{C}}$ is defined by the radius $R_{o}$ and the opening angle $\Theta_{0}$ (Fig. 2). Given that the geometry of the loaded state of the segment $\Omega_{2}^{\mathrm{C}}$ is known, the parameters $R_{o}$ and $\Theta_{0}$ can be obtained by solving an inverse identification problem. Actually, the problem is considered to be an optimisation problem where the vector of optimisation variables $\boldsymbol{u}=\left\{R_{o}, \Theta_{0}\right\}$ is supposed to minimise the corresponding objective function $\chi=\chi(\boldsymbol{u})$, which is defined so as to quantify the established difference between the desired and the computed response of the arterial segment in its loaded state. The desired response denotes the experimentally measured values of the selected quantities whereas the computed response refers to the same quantities predicted with the numerical model taking into account the optimisation vector $\boldsymbol{u}$.

In order to minimise (i) the error between the desired and computed stress state of the loaded configuration $\Omega_{2}^{\mathrm{C}}$, and (ii) the transmural gradient of the circumferential strain (which takes into account the uniform strain hypothesis), the objective function $\chi=\chi(\boldsymbol{u})$ is adequately structured, with functions $\chi_{\mathrm{P}}(\boldsymbol{u})$ and $\chi_{\lambda}(\boldsymbol{u})$ taking into account the respective effects:

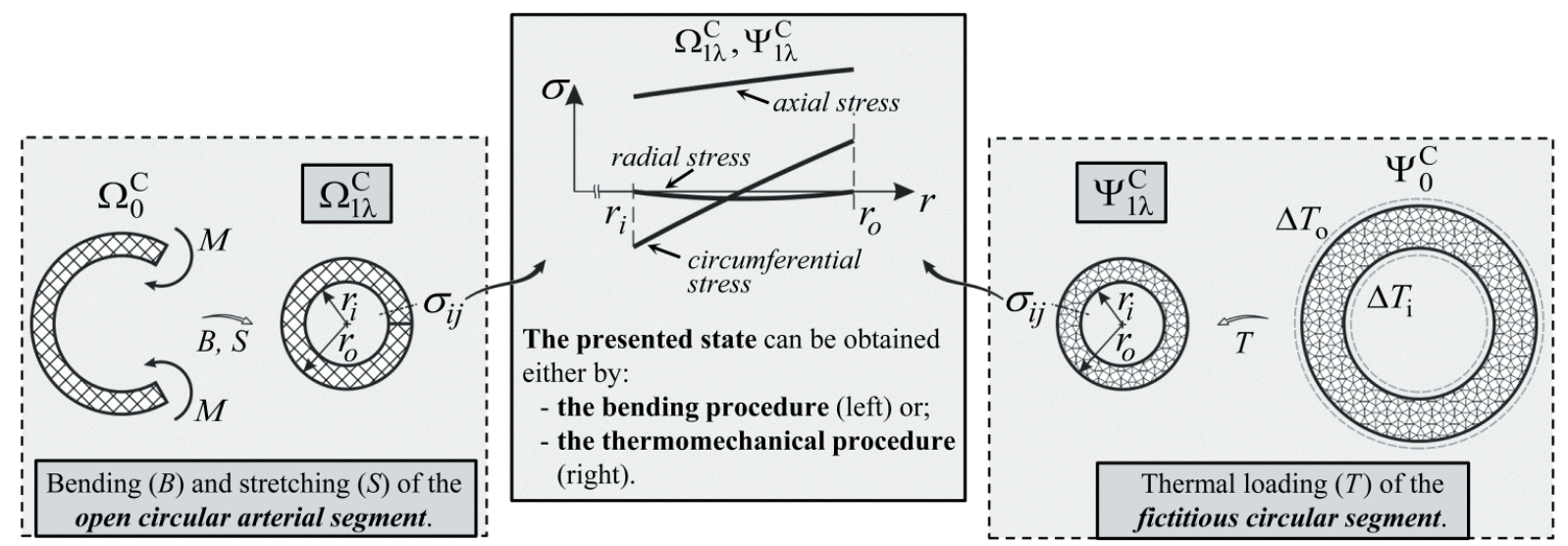

Fig. 3. Left-hand side of the figure: schematic representation of the stretched state of the circular arterial segment $\left(\Omega_{1 \lambda}^{\mathrm{C}}\right.$, Fig. 2); the same state (i.e., its geometry and stresses) can be obtained by thermally loading a closed tube (fictitious model), presented in the right-hand side of the figure 


$$
\begin{aligned}
\chi(\boldsymbol{u}) & =\chi_{\mathrm{P}}(\boldsymbol{u})+\chi_{\lambda}(\boldsymbol{u})= \\
& =\left(P_{\mathrm{c}}(\boldsymbol{u})-P\right)^{2}+g_{\lambda}\left(\lambda_{\phi}^{i}(\boldsymbol{u})-\lambda_{\phi}^{o}(\boldsymbol{u})\right)^{2} .
\end{aligned}
$$

Above, $P$ and $P_{c}$ are respectively the desired and the computed intraluminal pressures; $\lambda_{\phi}^{i}$ and $\lambda_{\phi}^{o}$ the circumferential stretches, computed at the inner and outer surface of the segment's wall; and $g_{\lambda}$ is the weighting factor.

\subsection{Determination of the Arterial Residual Stresses Using the Thermomechanical Analogy}

In this subsection, an alternative method for obtaining the stretched state of the circular arterial segment $\Omega_{1 \lambda}^{\mathrm{C}}$ (Fig. 3) using an analogy, based on the theory of thermoelasticity, is presented. Namely, from the theory of thermoelasticity it is known that the same deflection of, for instance, a cantilever beam can be obtained either by exposing the beam to an external bending moment at its free end or, alternatively, by adequately thermally loading the beam along its length. If we project this analogy to the modelling of the arterial response, this implies that the stretched state of the circular arterial segment $\Omega_{1 \lambda}^{\mathrm{C}}$, which is obtained by bending the open circular section $\Omega_{0}^{\mathrm{C}}$ and subsequent axial stretching, can equally be obtained by thermally loading an associated closed tube $\Psi_{0}^{\mathrm{C}}$. In Fig. 3, this is schematically outlined in the left and right box, respectively. The real significance of introducing the proposed thermomechanical analogy as a means to determine the residual stresses will be put forward in the subsequent sections, where the method will be adequately generalised and applied to a real artery's geometry.

The model considered in the described thermomechanical analogue needs to be properly defined, which is in fact the key step in the procedure of determining residual stresses. This model will be referred to in the sequel as the fictitious model because the material properties of the fictitious model, as will be shown, have nothing to do with the physical properties of the artery, and also because the thermal loading is fictitious with respect to the original mechanical model. Also, the boundary conditions have to be adequately changed in order to obtain the expected similarity of the mechanical response. The fictitious model will actually serve only as a mathematical tool to predict the arterial stretched state. The respective configurations of the fictitious model will be denoted with the symbol $\Psi$. The subscript supplementing $\Psi$ will specify the corresponding loading state of the fictitious model whereas the superscript of $\Psi$ will indicate the considered geometrical model. The superscript 'AM' will refer to the fictitious arterial model and ' $\mathrm{C}$ ' to the configurations of the fictitious circular segment.

Now, let us give a definition of the fictitious circular segment and the mathematical framework needed to compute its thermomechanical response. The fictitious circular segment, the role of which in the procedure of predicting the stretched state of a realistic arterial segment is schematically presented in the following subsection (see Fig. 5), is defined in the following way:

Geometry of the fictitious circular model in its zero-stress configuration $\Psi_{0}^{\mathrm{C}}$ (Fig. 4): The geometry of the fictitious model, which is assumed to be free of stresses, we defined based on the geometry of the corresponding arterial model in its loaded state (configuration $\Omega_{2}^{\mathrm{C}}$, see Fig. 2), thus: (i) the inner radius of the fictitious circular segment $\mathfrak{R}_{i}$ equals the inner radius of the corresponding circular arterial segment in its loaded state $r_{i}, \Re_{i}=r_{i}$; and (ii) the length of the fictitious model equals the length of the corresponding arterial model in its loaded state. The thickness of the fictitious model is denoted by $\delta_{f}$.

Material properties of the fictitious model: For the fictitious model a linearly elastic material is assumed. The material's behaviour needs, however, to be transversely isotropic with respect to the $z$ axis (Fig. 4). (Remark: A transversely isotropic material is characterised by a plane of isotropy at every point in the material.) Thus, in the fictitious model, the circumferential and axial stresses can be tuned independently by changing the material properties. This fact, as will be shown in the following sections, enables us to tune the material properties of the fictitious model in such a way that the stress distribution in the loaded state of the fictitious model $\Psi_{1 \lambda}^{\mathrm{C}}$ matches the stress distribution in the stretched state of the arterial segment $\Omega_{1 \lambda}^{\mathrm{C}}$. If the material behaviour of the fictitious model were isotropic this could not be achieved.

In cylindrical coordinates (Fig. 4) an elastic transversely isotropic material is defined by: "in plane" $(\mathrm{P})$ and transverse $(\mathrm{T})$ elastic moduli: $E_{r}=E_{\phi}=E_{P}$ and $E_{z}=E_{T}$; Poisson ratios: $v_{z r}=v_{z \phi}=v_{T P}$, $v_{r z}=v_{\phi z}=v_{P T}$; shear moduli: $G_{r \phi}=G_{P}, G_{r z}=G_{\phi z}=G_{T}$; and coefficients of the thermal expansion: $\alpha_{r}=\alpha_{\phi}=\alpha_{P}$, $\alpha_{z}=\alpha_{T}$ [25]. Moreover, the following relations hold: $v_{T P} / E_{T}=v_{P T} / E_{P}$ and $G_{P}=E_{P} / 2\left(1+v_{P}\right)$. The thermal properties of the fictitious model, i.e., the thermal conductivity and specific heat, are considered isotropic. 
Mechanical and thermal boundary conditions: Because the length of the fictitious model in the stressfree configuration $\Psi_{0}^{\mathrm{C}}$ already equals the length of $\Omega_{1 \lambda}^{\mathrm{C}}$, the model is prevented from deforming in the longitudinal direction when loaded. Consequently, the front and rear surfaces of the fictitious model are fixed in the axial direction (i.e., in the $z$ direction). In addition, the adiabatic boundary conditions are applied to these surfaces.

Thermal loading of the fictitious model: The fictitious model with the uniform initial temperature distribution $T(r)=0$ is exposed to a temperature change $\Delta T_{i}$ and $\Delta T_{o}$, applied, respectively, to its inner and outer surface (Fig. 4). Considering the given mechanical and thermal boundary conditions the stress distribution, corresponding to the established steady-state temperature field, is obtained.

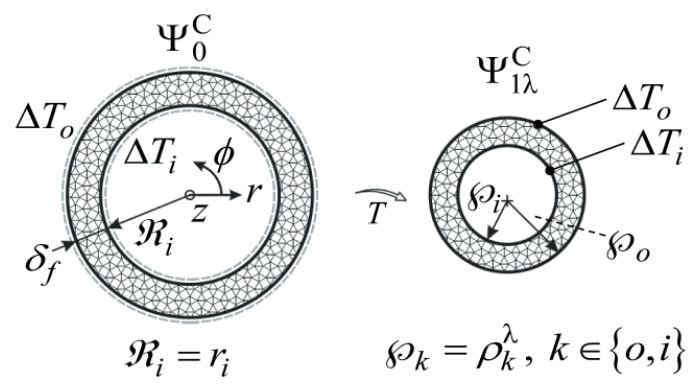

Fig. 4. Geometry and thermal loading of the fictitious model $\left(\Psi_{0}^{\mathrm{C}}\right)$; radii $r_{i}, \rho_{i}^{\lambda}$, and $\rho_{o}^{\lambda}$ are defined in Fig. 2; symbol $T$ represents thermal loading

Mathematical relations for computing the steadystate thermomechanical response of the fictitious circular segment are outlined next:

Steady-state thermal field: Taking into account the fact that the considered problem is a radial one, the steady-state heat equation, written in the cylindrical coordinates and with the omission of the internal heat generation term, is given as:

$$
\frac{1}{r} \frac{\mathrm{d}}{\mathrm{d} r}\left(r \frac{\mathrm{d} T}{\mathrm{~d} r}\right)=0
$$

where $r$ is the radius. Taking the applied temperature changes on the segment's surfaces, $T\left(\Re_{i}\right)=\Delta T_{i}$ and $T\left(\Re_{O}\right)=\Delta T_{o}$, as associate boundary conditions into account the following temperature distribution $T(r)$ is obtained by solving Eq. (12):

$$
T(r)=\Delta T_{i}+\frac{\Delta T_{o}-\Delta T_{i}}{\ln \left(\frac{\Re_{O}}{\mathfrak{R}_{i}}\right)} \ln \left(\frac{r}{\mathfrak{R}_{i}}\right),
$$

where $\Re_{i}=r_{i}$ and $\Re_{o}=\Re_{i}+\delta_{f}$ (Fig. 4).
Steady-state thermomechanical response: The stress state associated with the initial temperature distribution $T(r)=T_{a m b}=0$ being zero, the thermal stresses caused by the temperature change $T(r)$, Eq. (13), are governed by the generalised Hooke's law considering transversely isotropic material behaviour:

$$
\begin{gathered}
\varepsilon_{r r}=\frac{1}{E_{P}} \sigma_{r r}-\frac{v_{P}}{E_{P}} \sigma_{\phi \phi}-\frac{v_{T P}}{E_{T}} \sigma_{z z}+\alpha_{P} T, \\
\varepsilon_{\phi \phi}=-\frac{v_{P}}{E_{P}} \sigma_{r r}+\frac{1}{E_{P}} \sigma_{\phi \phi}-\frac{v_{T P}}{E_{T}} \sigma_{z z}+\alpha_{P} T, \\
\varepsilon_{z z}=-\frac{v_{P T}}{E_{P}} \sigma_{r r}-\frac{v_{P T}}{E_{P}} \sigma_{\phi \phi}+\frac{1}{E_{T}} \sigma_{z z}+\alpha_{T} T, \\
\varepsilon_{\phi z}=\frac{1}{G_{P}} \sigma_{\phi z}, \quad \varepsilon_{r z}=\frac{1}{G_{T}} \sigma_{r z}, \quad \varepsilon_{r \phi}=\frac{1}{G_{T}} \sigma_{r \phi},
\end{gathered}
$$

where $\varepsilon_{i j}$ and $\sigma_{i j}$ are the strain and the stress tensor. Next, the components of the strain tensor are expressed in terms of the radial and axial displacements $u(r)$ and $w(r)$ [26]:

$$
\begin{gathered}
\varepsilon_{r r}=\frac{\mathrm{d} u}{\mathrm{~d} r}, \quad \varepsilon_{\phi \phi}=\frac{u}{r}, \\
\varepsilon_{z z}=\frac{\mathrm{d} w}{\mathrm{~d} z}=e=\mathrm{const}, \\
\varepsilon_{r \phi}=\varepsilon_{r z}=\varepsilon_{\phi z}=0 .
\end{gathered}
$$

Using Eqs. (14) and (15), the following relationships between the stress tensor components and radial displacement $u(r)$ are obtained:

$$
\begin{gathered}
\sigma_{r r}=A_{\mathrm{P}} \frac{\mathrm{d} u}{\mathrm{~d} r}+A_{\mathrm{PT}} \frac{u}{r}+A_{\mathrm{TP}} e-\beta_{\mathrm{P}} T, \\
\sigma_{\phi \phi}=A_{\mathrm{PT}} \frac{\mathrm{d} u}{\mathrm{~d} r}+A_{\mathrm{P}} \frac{u}{r}+A_{\mathrm{TP}} e-\beta_{\mathrm{P}} T, \\
\sigma_{z z}=A_{\mathrm{TP}}\left(\frac{\mathrm{d} u}{\mathrm{~d} r}+\frac{u}{r}\right)+A_{\mathrm{T}} e-\beta_{\mathrm{T}} T, \\
\sigma_{r \phi}=\sigma_{r z}=\sigma_{\phi z}=0,
\end{gathered}
$$

where the constants $A_{\mathrm{P}}, A_{\mathrm{PT}}, A_{\mathrm{TP}}, A_{\mathrm{T}}, D, \beta_{\mathrm{P}}$ and $\beta_{\mathrm{T}}$ are defined as:

$$
\begin{gathered}
A_{\mathrm{P}}=\frac{E_{P}\left(1-v_{T P} v_{P T}\right)}{D}, A_{\mathrm{PT}}=\frac{E_{P}\left(v_{P}+v_{T P} v_{P T}\right)}{D}, \\
A_{\mathrm{TP}}=\frac{E_{P}\left(v_{T P}+v_{P} v_{T P}\right)}{D}, A_{\mathrm{T}}=\frac{E_{T}\left(1-v_{P}^{2}\right)}{D}, \\
D=1-2 v_{P} v_{T P} v_{P T}-2 v_{T P} v_{P T}-v_{P}^{2}, \\
\beta_{\mathrm{P}}=A_{\mathrm{P}} \alpha_{P}+A_{\mathrm{PT}} \alpha_{P}+A_{\mathrm{TP}} \alpha_{T}, \\
\beta_{\mathrm{T}}=2 A_{\mathrm{TP}} \alpha_{P}+A_{\mathrm{T}} \alpha_{T} .
\end{gathered}
$$


Next, by considering: (i) the expression for $\sigma_{r r}$ in (16) and (ii) the substitution of $\sigma_{\phi \phi}$, as specified in Eq. (16), in the equilibrium equation (Eq. (7)), the problem reduces to two first-order differential equations for the variables $u(r)$ and $\sigma_{r r}(r)$ [26], written as:

$$
\begin{aligned}
\frac{\mathrm{d} u}{\mathrm{~d} r}= & -\frac{A_{\mathrm{PT}}}{A_{\mathrm{P}}} \frac{u}{r}+\frac{\sigma_{r r}}{A_{\mathrm{P}}}-\frac{A_{\mathrm{TP}}}{A_{\mathrm{P}}} e+\frac{\beta_{\mathrm{P}}}{A_{\mathrm{P}}} T, \\
\frac{\mathrm{d} \sigma_{r r}}{\mathrm{~d} r} & =\left(A_{\mathrm{P}}-\frac{A_{\mathrm{PT}}^{2}}{A_{\mathrm{P}}}\right) \frac{u}{r^{2}}+\left(\frac{A_{\mathrm{PT}}}{A_{\mathrm{P}}}-1\right) \frac{\sigma_{r r}}{r}+ \\
& +\left(A_{\mathrm{TP}}-\frac{A_{\mathrm{PT}} A_{\mathrm{TP}}}{A_{\mathrm{P}}}\right) \frac{e}{r}+\left(\frac{A_{\mathrm{PT}}}{A_{\mathrm{P}}}-1\right) \frac{\beta_{\mathrm{P} T}}{r} .
\end{aligned}
$$

The natural boundary conditions for a traction-free hollow cylinder are: $\sigma_{r r}\left(\Re_{i}\right)=0$ and $\sigma_{r r}\left(\Re_{o}\right)=0$. The constant $e$ is zero $(e=0)$ because the segment is axially fixed. Once the system in Eq. (18) is solved taking into account the given boundary conditions, the remaining non-zero components of the stress tensor $\sigma_{\phi \phi}(r)$ and $\sigma_{z z}(r)$ can be obtained from the relations (Eq. (16)). The differential equations (Eq. (18)) were solved numerically using the finite difference method.

\subsubsection{Identification of the Unknown Model Parameters}

Up to now only a portion of the properties regarding the fictitious circular segment are defined. Actually, only the length and the inner radius $\mathfrak{R}_{i}$ of the segment's geometry in the zero-stress configuration $\Psi_{0}^{\mathrm{C}}$ have been specified while the thickness $\delta_{f}$ is unknown. Here, let us remember that the principal function of introducing the fictitious modelling is to match, by adequately choosing the respective model parameters, the stress distribution of the loaded state of the fictitious segment $\Psi_{1 \lambda}^{\mathrm{C}}$ with the stress distribution of the stretched state of the arterial segment $\Omega_{1 \lambda}^{\mathrm{C}}$. From the given theoretical framework, it follows that the mechanical response of the fictitious circular segment depends on: (i) the material properties, and (ii) the applied thermal loads, which may be considered to be unknowns to be determined in accordance with the expected objective.

The desired (targeting) state of the fictitious segment $\Psi_{1 \lambda}^{\mathrm{C}}$ is the stretched state of the associated circular arterial segment $\Omega_{1 \lambda}^{\mathrm{C}}$, i.e., its geometry and stress state. The parameters of the fictitious segment that need to be identified are: $E_{P}, \alpha_{P}, E_{T}, \alpha_{T}, \delta_{f}$, $\Delta T_{i}$, and $\Delta T_{o}$. The remaining material properties that also define the fictitious segment, namely the thermal conductivity $k$, specific heat $c$, density $\rho$, and Poisson's ratios $v_{P}$ and $v_{P T}$, either do not affect the steady-state thermomechanical response of the segment $(k, c, \rho)$ or are "interconnected" with some other material variables $\left(v_{P}, v_{P T}\right)$. Their values were therefore arbitrarily pre-set prior to the identification procedure to: $v_{P}=0.45, v_{P T}=0.1, k=100 \mathrm{~W} /(\mathrm{mK})$, $c=100 \mathrm{~J} /(\mathrm{kgK})$ and $\rho=2200 \mathrm{~kg} / \mathrm{m}^{3}$.

The objective function of the identification problem $\chi=\chi(v)$ is formulated to account for: (i) the relative error between the computed geometry (the fictitious segment) and desired geometry (the arterial segment), and (ii) the relative error between the computed and desired stress states; where $\boldsymbol{v}$ is the vector of optimisation variables $\boldsymbol{v}=\left(E_{P}, \alpha_{P}, E_{T}, \alpha_{T}, \delta_{f}, \Delta T_{i}, \Delta T_{o}\right)$. The equation reads:

$$
\begin{aligned}
\chi(\boldsymbol{v}) & =\sum_{m=r, \phi, z} \frac{1}{N_{m}} \sum_{k=1}^{N_{m}}\left(\frac{S_{\mathrm{ck}}^{m}(\boldsymbol{v})-S_{k}^{m}}{S_{k}^{m}}\right)^{2}+ \\
& +g_{\rho} \sum_{k=i, o}\left(\frac{\wp_{k}(\boldsymbol{v})-\rho_{k}^{\lambda}}{\rho_{k}^{\lambda}}\right)^{2},
\end{aligned}
$$

where the vectors $\boldsymbol{S}_{\mathrm{c}}^{m}$ and $\boldsymbol{S}^{m} ; m \in\{r, \phi, z\}$ are holding the computed and desired discrete values of the radial, circumferential, and axial stresses, respectively; and $N_{m} ; m \in\{r, \phi, z\}$ is the length of vector $\boldsymbol{S}^{m}$. (Note that in $\boldsymbol{S}^{m}$, only the non-zero values of the stresses are included.) The quantities $\wp_{k}$ and $\rho_{k}^{\lambda} ; k \in\{i, o\}$ are, respectively, the inner and outer radius of the fictitious (computed) and arterial (desired) segment, while $g_{\rho}$ is the weighting factor.

\subsection{Application of the Thermomechanical Analogy to a Realistic Arterial Segment}

Let us assume that the irregularly shaped, straight tube that is presented in the upper left box in Fig. 5 represents an arterial segment (AS) obtained by the medical imaging process. This subsection schematically presents how the thermomechanical analogy can be used to predict the stretched state of the observed AS $\Omega_{1 \lambda}^{\mathrm{AS}}$. The initial properties of the AS that have to be known for the procedure are: the AS material properties and the in vivo stretches $\lambda$ and $\Lambda$ (see Section 1).

First, the equivalent circular tube (segment) is defined based on the luminal cross-section area of the AS. The circular segment is then characterised as presented in Section 2.1 to determine its zerostress configuration $\Omega_{0}^{\mathrm{C}}$. Now that this configuration 


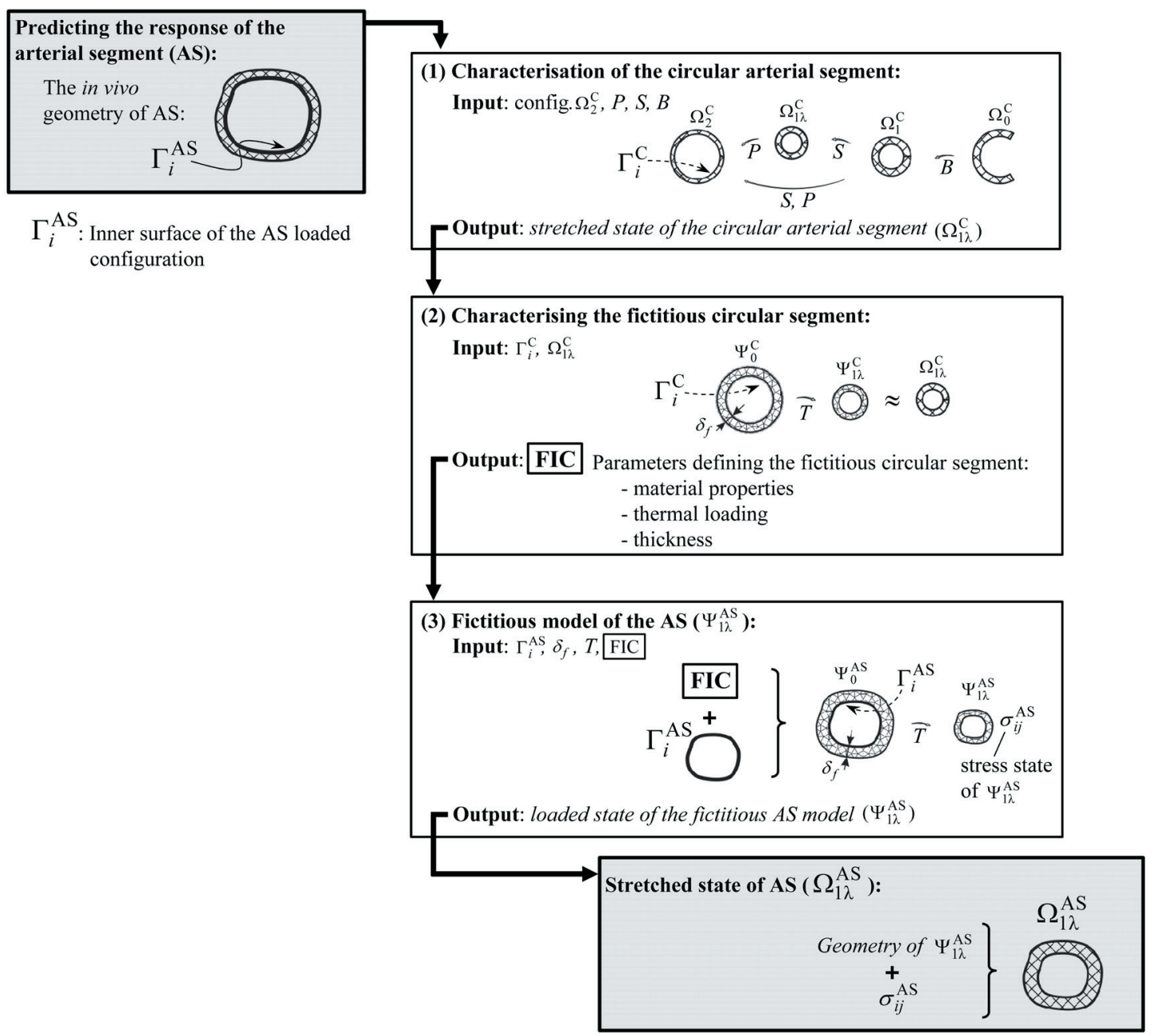

Fig. 5. Methodology that predicts the stretched state $\left(\Omega_{1 \lambda}^{\mathrm{AS}}\right)$ of a realistic arterial segment (AS)

is known, the stretched state of the circular arterial segment $\Omega_{1 \lambda}^{\mathrm{C}}$ can be computed.

Next, the thermomechanical analogy to predict the residual stresses is defined on the basis of the obtained $\Omega_{1 \lambda}^{\mathrm{C}}$ state. This is done by identifying the fictitious circular segment $\Psi_{0}^{\mathrm{C}}$ corresponding to the characterised circular arterial segment. The initial geometry of $\Psi_{0}^{\mathrm{C}}$ is defined based on the surface $\Gamma_{i}^{\mathrm{C}}$ (see Fig. 5) whereas the thickness and material properties of $\Psi_{0}^{\mathrm{C}}$ should be identified so that the loaded state of the fictitious circular segment $\Psi_{1 \lambda}^{\mathrm{C}}$ resembles the stretched state of the circular arterial segment $\Omega_{1 \lambda}^{\mathrm{C}}$.

So far in this subsection, the stretched state of the AS has been quantified on the basis of a simplified AS geometry assumed to have the form of a circular tube. Based on this simplification, the parameters that characterise the fictitious circular segment $\Psi_{0}^{\mathrm{C}}$ have been obtained. Next, the fictitious AS model $\Psi_{0}^{\mathrm{AS}}$ needs to be defined. This is done by applying the identified parameters of the fictitious circular segment (represented as FIC in Fig. 5) to the luminal surface of the AS, i.e., the surface $\Gamma_{i}^{\mathrm{AS}}$. With thus defined $\Psi_{0}^{\mathrm{AS}}$ state, the thermomechanical response of the fictitious AS $\left(\Psi_{1 \lambda}^{\mathrm{AS}}\right)$ can be computed. Note that in general this computation, i.e., solving the thermomechanical problem of the fictitious AS model, cannot be performed analytically because of the irregular geometry of the AS model. An approximate method, such as FEM, needs to be considered.

Finally, based on the results of the $\Psi_{1 \lambda}^{\mathrm{AS}}$ state the AS stretched state $\Omega_{1 \lambda}^{\mathrm{AS}}$ is approximated - note that $\Omega_{1 \lambda}^{\mathrm{AS}}$ is just defined based on the state $\Psi_{1 \lambda}^{\mathrm{AS}}$ and thus $\Omega_{1 \lambda}^{A S}$ still includes the nonlinearities of the arterial mechanical response. The geometry of the $\Omega_{1 \lambda}^{\mathrm{AS}}$ state 
equals the geometry of the $\Psi_{1 \lambda}^{\mathrm{AS}}$ state, and the stresses of the $\Omega_{1 \lambda}^{\mathrm{AS}}$ state equal the stresses of the $\Psi_{1 \lambda}^{\mathrm{AS}}$ state.

\section{APPLICATION OF THE THERMOMECHANICAL ANALOGY TO THE ARTERIAL MODEL}

The methodology for determining the residual stresses in the arterial segment, which were discussed thoroughly in the previous section, is generalised in this section to enable a prediction of the arterial response based on the in vivo arterial data. The individual steps of the methodology are demonstrated considering the AM, defined in Section 1 (Fig. 1).

To give an overview of the methodology, let us consider the flowchart presented in Fig. 6: (i) the starting data, needed for a prediction of the AM stretched state $\Omega_{1 \lambda}^{\mathrm{AM}}$, are the AM loaded geometry, the corresponding intraluminal pressure, the in vivo stretch $\lambda$, and the material properties of the AM. (ii) The stretched state of the AM is quantified based on characterising the mechanical response of two idealised (circular) arterial segments, corresponding to the arterial smallest (m) and largest (M) luminal crosssectional area (see Fig. 1). (iii) The fictitious arterial model (FAM), i.e., the fictitious model of the AM, is defined next. This is done by defining the fictitious circular segments for the aforementioned circular arterial segments. The identified parameters of the fictitious circular segments are then interpolated/ extrapolated over the AM luminal cross-sectional area $A(z)$ and applied to the AM luminal surface $\Gamma_{i}^{\mathrm{AM}}$. In this way, the FAM in its zero-stress state $\Psi_{0}^{\mathrm{AM}}$ is obtained. Its loaded state $\Psi_{1 \lambda}^{\mathrm{AM}}$ is then predicted by solving the corresponding steady-state thermomechanical problem. (iv) Finally, the AM stretched state $\Omega_{1 \lambda}^{\mathrm{AM}}$ is defined based on the obtained FAM $\Psi_{1 \lambda}^{\mathrm{AM}}$ state (i.e., based on the $\Psi_{1 \lambda}^{\mathrm{AM}}$ geometry and stress state). Further on, the loaded state of the AM $\Omega_{2}^{\mathrm{AM}}$, as well as the AM zero-stress state $\Omega_{0}^{\mathrm{AM}}$, are predicted. The individual steps of the methodology are presented in more detail in the following subsections.

\subsection{Quantification of the AM Stretched State}

The quantification of the AM stretched state is performed based on the observation of two idealised (circular) arterial segments that refer to the arterial smallest (m) and largest (M) luminal cross-sectional area, see Fig. 1b. The respective segments' geometry is obtained from the AM cross-sectional area $A(z)$ on the area equivalence basis:

$$
A(z)=\pi a(z) b(z)=\pi\left(\tilde{r}_{i}(z)\right)^{2},
$$

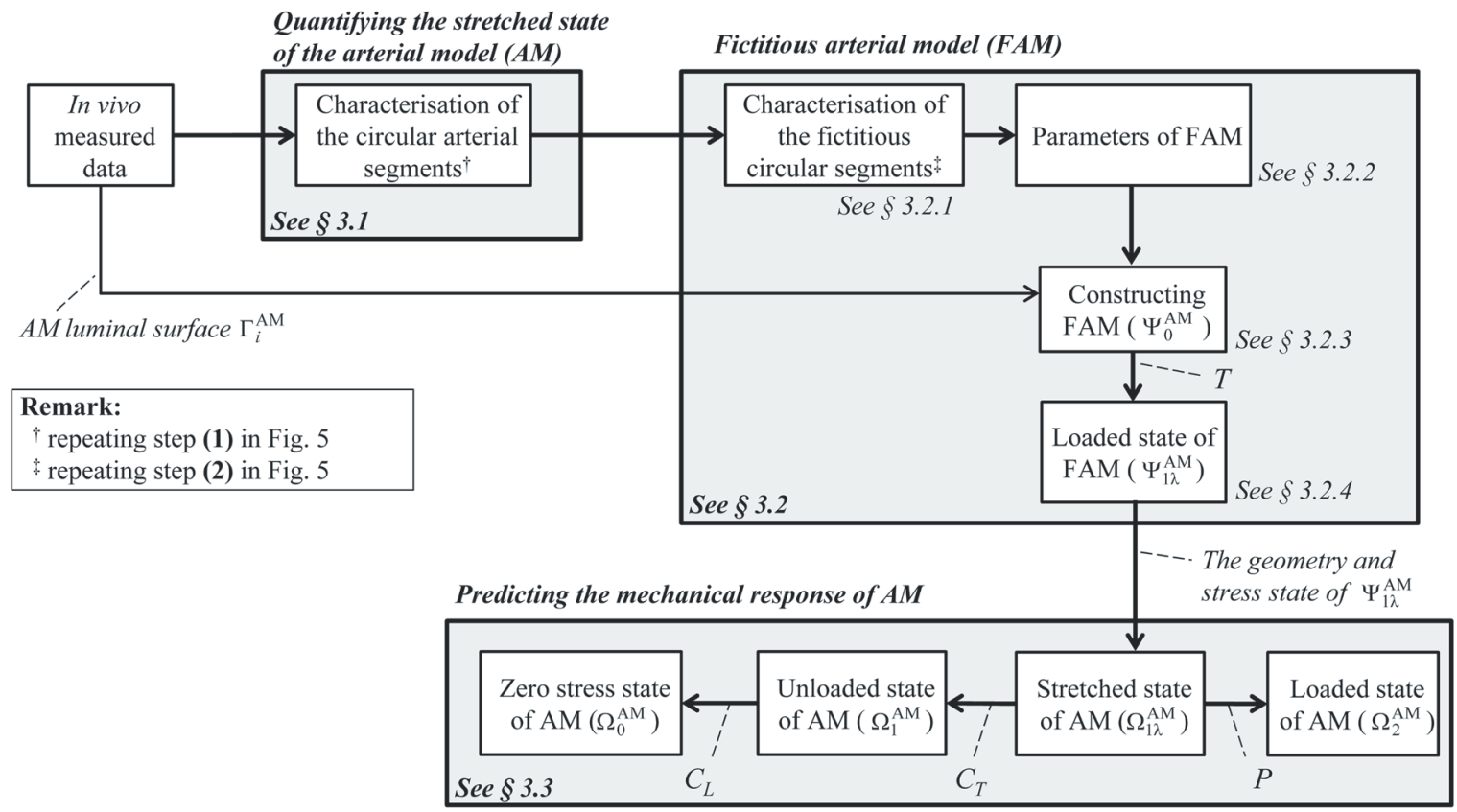

Fig. 6. The methodology that predicts the mechanical response of the artery based on the arterial in vivo geometry (i.e., without the arterial zero-stress state being known); the symbols $P, T, C_{T}$, and $C_{L}$ represent pressurising, thermal loading, transversal cut, and longitudinal cut, respectively 
from where the equivalent radius $\tilde{r}_{i}(z)$, see $r_{i}$ Fig. 2, is computed, and in particular, $\tilde{r}_{i}^{(\mathrm{k})}=\sqrt{A^{(\mathrm{k})} / \pi}$; $k \in\{\mathrm{m}, \mathrm{M}\}$ for segment (m) and (M), respectively. Next, the zero-stress state configurations of both segments, (m) and (M), are separately identified (see Section 2.1). The identification results are tabulated in Table 1, where $R_{o}^{(k)}$ and $\Theta_{0}^{(k)} ; k \in\{\mathrm{m}, \mathrm{M}\}$ are respectively the outer radius and the opening angle for the segment $(\mathrm{m})$ and $(\mathrm{m})$.

With the zero-stress state of both segments determined, their stretched states, designated as $\Omega_{1 \lambda}^{(\mathrm{m})}$ and $\Omega_{1 \lambda}^{(\mathrm{M})}$ for the segment $(\mathrm{m})$ and $(\mathrm{M})$, respectively, can be computed.

Table 1. The identified properties of the circular arterial segments

\begin{tabular}{lccc}
\hline Segment: $k$ & $(\mathrm{~m})$ & $(\mathrm{M})$ & $(\tilde{\mathrm{m}})$ \\
\hline$R_{O}^{(k)}[\mathrm{mm}]$ & 4.45 & 5.34 & 4.9 \\
\hline$\Theta_{0}^{(k)}\left[^{\circ}\right]$ & 132.89 & 129.94 & 131.34 \\
\hline
\end{tabular}

\subsection{Fictitious Arterial Model}

As discussed in Section 2.2, the AM stretched state $\Omega_{1 \lambda}^{\mathrm{AM}}$ can be predicted by defining an associated fictitious arterial model, designated as FAM and solving the corresponding thermomechanical problem. The advantage of this approach is that the arterial response is predicted without the arterial zerostress state being known. How to define the FAM is discussed in detail in the following subsections.

\subsubsection{Thermomechanical Characterisation of the Fictitious Circular Segments}

The thermomechanical analysis of the FAM is basically defined by the characterisation of the fictitious circular segments corresponding to the circular arterial segments (m) and (M), as presented in step 2 in Fig. 5. Note that in this way, essentially, the properties of the FAM for its location $A^{(\mathrm{m})}$ and $A$ (M), see Fig. 1, are defined.

The variables that need to be identified in order to characterise both fictitious segments (see Section 2.3) are: the material properties, the thicknesses $\delta_{f}^{(k)}$, the thermal loadings $\Delta T_{i}^{(k)}$, and $\Delta T_{o}^{(k)} ; k \in\{\mathrm{m}, \mathrm{M}\}$ for segment $(\mathrm{m})$ and $(\mathrm{M})$, respectively. During the identification of the unknowns, the material properties of both segments were initially assumed to be equal because they represent the material properties of the FAM. However, what was found is that in order for the stresses of both fictitious segments to correspond to the stresses of the associated arterial segments, the coefficient of thermal expansion $\alpha_{T}$ has to be identified separately for each segment.

Because of a relatively large number of unknowns, the identification procedure was performed in the following way. First, the identification of the parameters defining the fictitious segment $(\mathrm{m})$ (see Section 2.2.1.) is performed. The unknowns to be determined: thickness $\delta_{f}^{(\mathrm{m})}$, material constants $E_{P}$, $\alpha_{P}, E_{T}$ and $\alpha_{T}^{(\mathrm{m})}$, and thermal loadings $\Delta T_{i}^{(\mathrm{m})}$ and $\Delta T_{o}^{(\mathrm{m})}$, are identified by targeting the $\Omega_{1 \lambda}^{(\mathrm{m})}$ state of the circular arterial segment $(\mathrm{m})$. Next, the identification of the fictitious segment (M) is performed by adopting the material properties identified for the segment $(\mathrm{m})$.

The remaining variables to be determined: $\delta_{f}^{(\mathrm{M})}$, $\Delta T_{i}^{(\mathrm{M})}, \Delta T_{o}^{(\mathrm{M})}$, and $\alpha_{T}^{(\mathrm{M})}$, are obtained by targeting the $\Omega_{1 \lambda}^{(\mathrm{M})}$ state of the circular arterial segment $(\mathrm{M})$. The result of the identification is given in Table 2 .

Table 2. The identified properties of the fictitious circular segments

\begin{tabular}{llcl}
\hline \multicolumn{1}{c}{ Segment: $k$} & & $(\mathrm{~m})$ & $(\mathrm{M})$ \\
\hline$\Delta T_{i}^{(k)}$ & $\left.{ }^{\circ}\right]$ & 1.0059 & 1.069 \\
\hline$\Delta T_{o}^{(k)}$ & $\left.{ }^{\circ}\right]$ & 1.255 & 1.295 \\
\hline$\delta_{f}^{(k)}$ & {$[\mathrm{mm}]$} & 0.6931 & 0.71 \\
\hline$\alpha_{T}^{(k)}$ & {$[1 / \mathrm{K}]$} & -0.05 & -0.048 \\
\hline \hline$E_{P}$ & {$[\mathrm{kPa}]$} & 299.29 & \\
\hline$E_{T}$ & {$[\mathrm{kPa}]$} & 299.29 & \\
\hline$\alpha_{P}$ & {$[1 / \mathrm{K}]$} & -0.1388 &
\end{tabular}

3.2.2 Thermomechanical Characterisation of the Fictitious Arterial Model

As mentioned, the parameters of the FAM are defined based on the parameters identified for the fictitious circular segments $(\mathrm{m})$ and $(\mathrm{M})$. The parameters that are common to both fictitious segments are also assumed constant in the FAM. These are: $E_{P}, \alpha_{P}, E_{T}$, $G_{P}, G_{T}, v_{P}, v_{P T}, k, c$ and $\rho$ (see Section 2.2). However, some parameters of the fictitious segments, namely their thickness $\delta_{f}$, thermal loadings $\Delta T_{i}$ and $\Delta T_{o}$, and the coefficient of thermal expansion $\alpha_{T}$, are not the same in both fictitious segments. We will refer to these variables as the varying variables because they depend on the cross-sectional area of the fictitious circular segments. Note that the cross-sectional area of the FAM also varies - from $A^{(\mathrm{m})}$ to $A^{(\mathrm{M})}$. Therefore, 
in order to apply the varying variables to the FAM, their values need to be generalised (interpolated) over the cross-sectional area $A(z)$ of the FAM.

In this paper, a linear interpolation of the varying variables across the $A(z)$, i.e., from $A^{(\mathrm{m})}$ to $A^{(\mathrm{M})}$, is adopted. For example, a linear interpolation of the variable $\alpha_{T}^{\mathrm{AM}}$ (where the superscript "AM" refers to the interpolated variable) as a function of $A(z)$ can be written as:

$$
\begin{aligned}
\alpha_{T}^{\mathrm{AM}}(A) & =\alpha_{T}^{(\mathrm{m})} \Upsilon_{\mathrm{m}}(A)+ \\
& +\alpha_{T}^{(\mathrm{M})} \Upsilon_{\mathrm{M}}(A)=\alpha_{T}^{\mathrm{AM}}(z),
\end{aligned}
$$

where $\quad \alpha_{T}^{(\mathrm{m})}=\alpha_{T}\left(A^{(\mathrm{m})}\right) \quad$ and $\quad \alpha_{T}^{(\mathrm{M})}=\alpha_{T}\left(A^{(\mathrm{M})}\right)$, and the shape functions $\Upsilon_{\mathrm{m}}(A)$ and $\Upsilon_{\mathrm{M}}(A)$ are defined $\quad$ as: $\quad \Upsilon_{\mathrm{m}}(A)=1-\frac{A-A^{(\mathrm{m})}}{A^{(\mathrm{M})}-A^{(\mathrm{m})}}=\Upsilon_{\mathrm{m}}(z)$ and $\Upsilon_{\mathrm{M}}(A)=\frac{A-A^{(\mathrm{m})}}{A^{(\mathrm{M})}-A^{(\mathrm{m})}}=\Upsilon_{\mathrm{M}}(z)$. Note that $\alpha_{T}^{\operatorname{AM}_{(A)}}$ (as well as $\Upsilon_{\mathrm{m}}(A)$ and $\left.\Upsilon_{\mathrm{M}}(A)\right)$ is essentially a function of the coordinate $z$, thus $\alpha_{T}^{\mathrm{AM}_{(z)}}$ because $A=A(z)$ (see Fig. 1). The same interpolation approach is used to define the remaining varying variables: $\delta_{f}^{\mathrm{AM}}(z), \Delta T_{i}^{\mathrm{AM}}(z)$ and $\Delta T_{o}^{\mathrm{AM}}(z)$. Their variations as a function of the coordinate $z$ are plotted in Fig. 7.

Remark: For the purpose of defining the properties of the FAM, it would be possible to characterise more than two circular arterial segments. For these segments, the corresponding fictitious segments could be obtained and characterised, thus allowing for a higher-order interpolation used in the
FAM characterisation. However, as shown in Section 4.1.1, the linear interpolation, which is used to define the FAM properties, is sufficient.

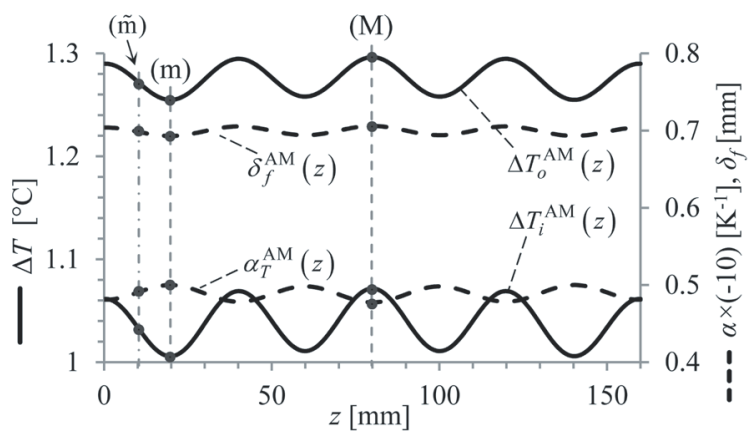

Fig. 7. Variations of the FAM properties: $\alpha_{T}^{\mathrm{AM}}(z), \delta_{f}^{\mathrm{AM}}(z)$, $\Delta T_{i}^{\mathrm{AM}}(z)$, and $\Delta T_{o}^{\mathrm{AM}}(z)$ (see Eq. (21)), along the coordinate $z$

\subsubsection{Construction of the Fictitious Arterial Model (FAM) Considering in vivo Measured Data}

Based on the characterisation of the FAM parameters that were determined in previous subsections under the assumption of idealised circular arterial segments, it is now possible to construct a computational model of the FAM taking in vivo measured data into account. Accordingly, the geometry of the FAM is constructed based on the inner surface of the $\mathrm{AM} \Gamma_{i}^{\mathrm{AM}}$ (Fig. 1), as shown in Fig. 8, considering its variation $\Gamma_{i}^{\mathrm{AM}}$ along the $z$ direction. As discussed, we define the inner surface of the FAM to be the same as the inner (luminal) surface of the associated AM, whereas the outer surface of the FAM is obtained by applying the thickness $\delta_{f}^{\mathrm{AM}}(z)$ (Fig. 7) to the surface $\Gamma_{i}^{\mathrm{AM}}$.

Constructing the fictitious arterial model $\Psi_{0}^{\mathrm{AM}}$

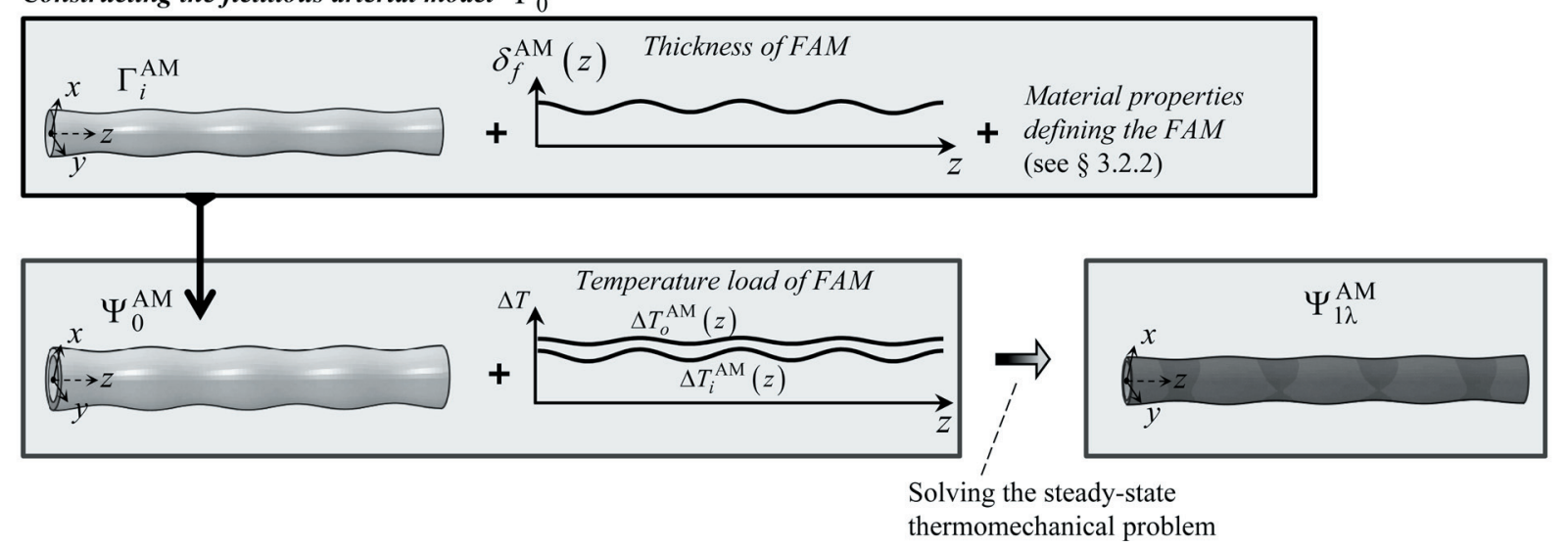

Fig. 8. Constructing the fictitious arterial model 
Note that the thickness of the FAM is assumed to be constant in the circumferential direction of the model while changing in the $z$ direction.

As assumed, the material behaviour of the FAM is transversely isotropic with respect to the $z$ axis. Some of the material properties are constant (see Table 2) whereas $\alpha_{T}^{\mathrm{AM}}(z)$ is given in Fig. 7. The remaining material parameters that are not presented in Table 2 are given in Section 2.2.1.

\subsubsection{Loaded State of the Fictitious Arterial Model}

In order to evaluate the residual stresses of the AM, the above-defined FAM is thermally loaded by the temperature changes $\Delta T_{i}^{\mathrm{AM}}(z)$ and $\Delta T_{o}^{\mathrm{AM}}(z)$ applied respectively on the inner and outer surfaces of the model (Fig. 8). Note that both $\Delta T_{i}^{\mathrm{AM}}(z)$ and $\Delta T_{o}^{\mathrm{AM}}(z)$ (Fig. 7) are assumed to be constant in the circumferential direction of the model, while changing in the $z$ direction. The stress distribution in the loaded state of the FAM $\Psi_{1 \lambda}^{\mathrm{AM}}$, obtained by solving the given thermomechanical problem with associated mechanical and thermal boundary conditions (see Section 2.2), yields an approximation of the residual stresses distribution in the AM and its configuration.

\subsection{Modelling the Mechanical Response of the Arterial Model with Residual Stresses Considered}

The geometry and the stresses of the loaded state $\Psi_{1 \lambda}^{\mathrm{AM}}$ of the FAM represent an approximation to the geometry and stresses of the arterial model in its stretched state $\Omega_{1 \lambda}^{\mathrm{AM}}$. The computational model of the AM stretched state $\Omega_{1 \lambda}^{\mathrm{AM}}$ is thus specified by adopting the geometry of the $\Psi_{1 \lambda}^{\mathrm{AM}}$ configuration and the given arterial material behaviour. For the initial stress state of the thus-defined model, representing, actually, the residual stresses in the artery, the stresses from $\Psi_{1 \lambda}^{\mathrm{AM}}$ are adopted whereas the stretches corresponding to the $\Omega_{1 \lambda}^{\mathrm{AM}}$ configuration with the initial stresses considered are obtained by taking into account the arterial material model, Eq. (1).

From the arterial stretched state all the remaining arterial states can be predicted (see the lower grey box in Fig. 6). The loaded state $\Omega_{2}^{\mathrm{AM}}$ of the AM is obtained by inflating the AM from its stretched state. The $\mathrm{AM}$ unloaded state $\Omega_{1 \lambda}^{\mathrm{AM}}$ is predicted by releasing the axial stretch $\lambda$ from the AM stretched state, whereas the approximation of the AM zero-stress state $\Omega_{0}^{\mathrm{AM}}$ is obtained by further releasing the constraints on one of the AM symmetry surfaces (Fig. 2).
Remark: The reason why the arterial stretched state $\Omega_{1 \lambda}^{\mathrm{AM}}$ is predicted with the fictitious model, and not, for instance, by considering the arterial unloaded state $\Omega_{1}^{\mathrm{AM}}$, is the following: normally, the goal of the arterial characterisation is to predict the arterial loaded state. Therefore it is more convenient to predict the arterial loaded state from the arterial stretched state given that in between both states there is only the inflation process (see Fig. 2). For example, by predicting the arterial loaded state from the arterial unloaded state, the artery first needs to be stretched for $\lambda$ in order to obtain its stretched state, and can only later be inflated.

\section{RESULTS, VALIDATION OF THE METHODOLOGY AND DISCUSSION}

In this section, the developed methodology of determining the arterial response based on the in vivo measured arterial data and taking the residual stresses into account is applied in the analysis of the given case study (Fig. 1). In the analysis, regardless of whether the computation applies to the real or the fictitious model, the computations that relate to the circular models are performed using the finite difference method while the FEM is used, because of the complexity of respective model geometries, for the computations related to the arterial models.

First, after giving a brief description of the numerical model in Section 4.1, the results for the circular arterial and fictitious segments are presented in Section 4.2. The results regarding the arterial response are presented in Sections 4.3.1 and 4.3.2 for the AM stretched and loaded state, respectively. Finally, at the end of the section, the methodology is validated based on the prediction of the AM zerostress state.

\subsection{FEM Modelling}

Taking into consideration that the geometry of the FAM loaded state $\Psi_{1 \lambda}^{\mathrm{AM}}$ configuration may be considered to be an approximation to the geometry of the AM stretched state $\Omega_{1 \lambda}^{\mathrm{AM}}$ configuration, only one single FEM discretisation is needed for all of the computations regardless of whether the computation applies to the real or the fictitious model.

The FEM model is constructed based on the geometry of the FAM in its stress-free configuration $\Psi_{0}^{\text {AM }}$ (Fig. 8) taking into account the data specified in Section 3.2.3. In principle, because the considered case study (Fig. 1) is symmetric with respect to two perpendicular planes (note that the cross section of the 
AM is elliptical), only one quarter of the model could be considered for computations. However, in order for the computation of the AM zero-stress state $\Omega_{2}^{\mathrm{AM}}$ to also be performed (see Fig. 2), half of the model is considered for the FEM discretisation with the $x-z$ plane being the plane of symmetry. The FEM model is built of 17640 eight-node brick elements with displacement and temperature degrees of freedom.

The conceived FEM model is used first for the computation of the FAM loaded state $\Psi_{1 \lambda}^{\mathrm{AM}}$. For this purpose, a linearly elastic, transversely isotropic behaviour of the material is assumed taking the data specified in Section 3.2.3 into account. The thermomechanical analysis is performed using the commercial FEM software Abaqus/Standard [27] under prescribed boundary and loading conditions. The front and rear surfaces of the model are fixed in the $z$ direction whereas they are free to move in the $x-y$ plane. On the same surfaces, the adiabatic thermal conditions are applied. Moreover, proper mechanical and thermal conditions are applied on the symmetry surfaces (see Section 3.2.4).

The stresses of the FAM loaded state $\Psi_{1 \lambda}^{\mathrm{AM}}$ represent an approximation of the residual stresses in the artery. The computational model of the AM stretched state $\Omega_{1 \lambda}^{\mathrm{AM}}$ is thus specified by adopting the geometry of the $\Psi_{1 \lambda}^{\mathrm{AM}}$ configuration and related stresses as initial stresses, and, importantly, taking the given arterial material behaviour into account, Eq. (1). Clearly, the stretches corresponding to the $\Omega_{1 \lambda}^{\mathrm{AM}}$ configuration are readily computed considering the constitutive relations and the initial stresses. When the thus-defined computational model $\Omega_{1 \lambda}^{\mathrm{AM}}$ is inflated with the intraluminal pressure $P$, the loaded state $\Omega_{2}^{\mathrm{AM}}$ is computed. If, on the contrary, the constraints on the axial displacement are removed and the

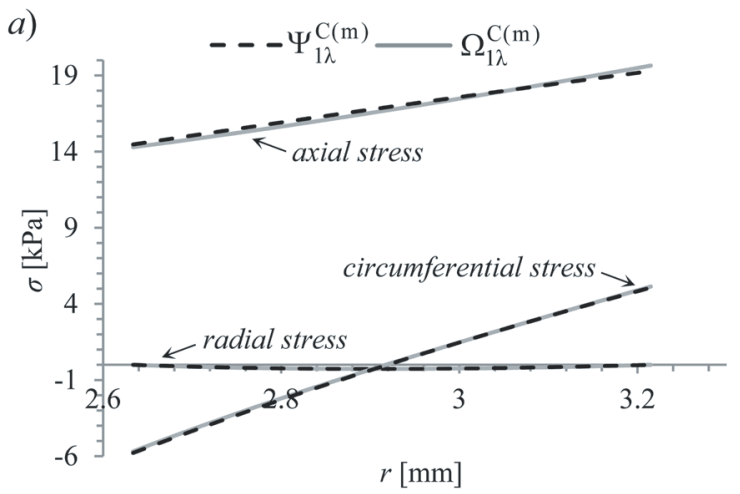

Fig. 9. Stresses computed for the $\Omega_{1 \lambda}^{\mathrm{C}(\mathrm{m})}$ and $\Omega_{1 \lambda}^{\mathrm{C}(\mathrm{M})}$ states of the circular arterial segments (full line), and their comparison with the stresses obtained for the fictitious circular segments $\Psi_{1 \lambda}^{\mathrm{C}(\mathrm{m})}$ and $\Psi_{1 \lambda}^{\mathrm{C}(\mathrm{M})}$ (broken line); the results for the segment ( $m$ ) are given in a) whereas $b$ ) shows the results for the segment (M) 
obtained with the fictitious segments (m) and (M), respectively. In the next subsection, we shall assess the used linear interpolation that was applied to interpolate the properties of the fictitious segments with the purpose of defining the FAM (see Section 3.2.2).

\subsubsection{The Adequacy of the Considered Interpolation}

The variables $\alpha_{T}^{\mathrm{AM}}(z), \delta_{f}^{\mathrm{AM}}(z), \Delta T_{i}^{\mathrm{AM}}(z)$ and $\Delta T_{o}^{\mathrm{AM}}(z)$ were obtained by linearly interpolating the corresponding variables that have been determined for the fictitious segments (m) and (M) (see Section 3.2.2). The adequacy of this approximation is assessed by characterizing an arterial segment, denoted by $(\tilde{\mathrm{m}})$, with the equivalent radius $\tilde{r}_{i}^{(\tilde{\mathrm{m}})}=\left(\tilde{r}_{i}^{(\mathrm{m})}+\tilde{r}_{i}^{(\mathrm{M})}\right) / 2$. For the segment $(\tilde{\mathrm{m}})$, its stretched state $\Omega_{1 \lambda}^{\mathrm{C}(\tilde{\mathrm{m}})}$ is identified and then compared to the stretched state, obtained through defining an associated fictitious segment ( $\Psi_{1 \lambda}^{\mathrm{C}(\tilde{\mathrm{m}})}$ state).

The parameters of the fictitious segment ( $\tilde{\mathrm{m}})$ are obtained by considering the data in Fig. 7 and Table 2. The loaded state of the fictitious segment $\left(\Psi_{1 \lambda}^{\mathrm{C}(\tilde{\mathrm{m}})}\right)$ is computed as presented in Section 2.2.
To determine the stretched state of the arterial segment $(\tilde{\mathrm{m}})$, its zero-stress configuration needs to be identified as presented in Section 2.1. The identified values of the unknowns $R_{o}^{(\tilde{\mathrm{m}})}$ and $\Theta_{0}^{(\tilde{\mathrm{m}})}$ are given in Table 1. The computed stresses of the arterial segment $\left(\Omega_{1 \lambda}^{\mathrm{C}(\tilde{\mathrm{m}})}\right)$ and the fictitious segment $\left(\Psi_{1 \lambda}^{\mathrm{C}(\tilde{\mathrm{m}})}\right)$ are presented in Fig. 10.

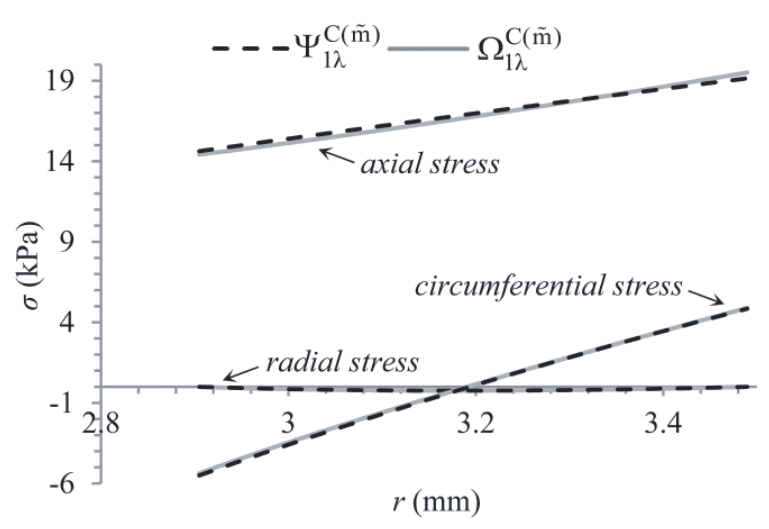

Fig. 10. The calculated stresses of the $\Omega_{1 \lambda}^{\mathrm{C}(\tilde{\mathrm{m}})}$ state of the circular artery segment ( $\tilde{\mathrm{m}})$ (full line), compared to stresses of the $\Psi_{1 \lambda}^{\mathrm{C}(\tilde{\mathrm{m}})}$ state, computed with the associated fictitious circular segment (broken line) a)

a)
$\begin{aligned} & \sigma_{\phi \phi}[\mathrm{kPa}] \\ & \square^{6.4} \\ & 2 \\ & 0 \\ & -2 \\ & -3 \\ & -6.15\end{aligned}$

b)
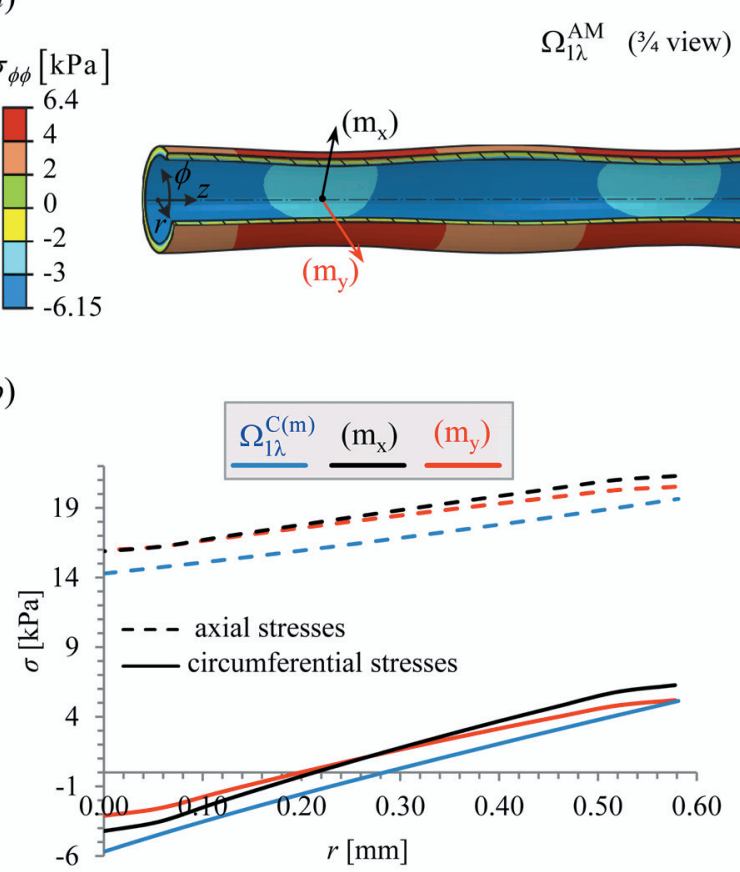

$\left(\mathrm{m}_{\mathrm{y}}\right)^{\prime}$
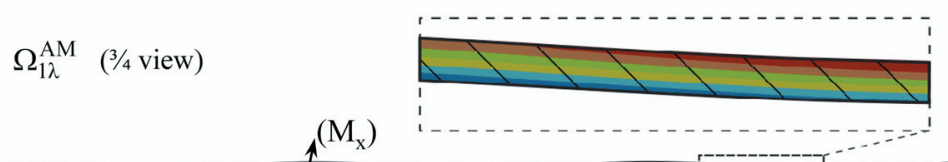

$\left(\mathrm{M}_{\mathrm{x}}\right)$

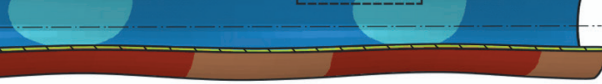

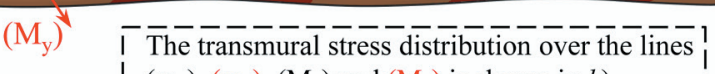

$\left(\mathrm{m}_{\mathrm{x}}\right),\left(\mathrm{m}_{\mathrm{y}}\right),\left(\mathrm{M}_{\mathrm{x}}\right)$ and $\left(\mathrm{M}_{\mathrm{y}}\right)$ is shown in $\left.b\right)$.

Fig. 11. The predicted AM loaded state $\left(\Omega_{2}^{\mathrm{AM}}\right)$ : a) $3 \mathrm{D}$ view and b) the transmural distribution of the circumferential and axial stresses at the AM positions $\left(\mathrm{m}_{\mathrm{x}}\right),\left(\mathrm{m}_{\mathrm{y}}\right),\left(\mathrm{M}_{\mathrm{x}}\right)$, and $\left(\mathrm{M}_{\mathrm{y}}\right)$; for their location see Fig. $11 \mathrm{a}$ 
Despite a slight discrepancy between the axial stresses (Fig. 10), the agreement between the obtained arterial and fictitious state of the segment $(\tilde{\mathrm{m}})$ is excellent. Based on these results, it is possible to conclude that the first-order interpolation, which is used to interpolate the properties of the fictitious segments, is justified.

\subsection{The Predicted Mechanical Response of AM}

\subsubsection{AM Stretched State}

The circumferential stresses $\sigma_{\phi \phi}$ of the predicted AM stretched state $\left(\Omega_{1 \lambda}^{\mathrm{AM}}\right)$ are presented in Fig. 11a. The transmural variation of the $\sigma_{\phi \phi}$ shows the bending distribution (see the zoomed-in detail along the wall thickness), as was also observed for the circular arterial segments. In contrast to the straight circular arterial segment, the stresses in the AM vary along the AM length because of the varying AM cross-sectional area. The transmural distribution of the stresses at the AM positions (m) and (M) are shown in Fig. 11b (red and black curves). In the figure, the stresses are presented at the position of the AM cross-sectional $x$ and $y$ axis. Note that the stresses at position (m) are higher on the $\left(\mathrm{m}_{\mathrm{x}}\right)$ axis compared to the values on the $\left(\mathrm{m}_{\mathrm{y}}\right)$ axis. At position $(\mathrm{M})$, on the other hand, the opposite is observed. This is interesting because the $x$ axis represents the cross-sectional semi-minor axis at both position (m) as well as at position (M) (see Fig. 1). The reason for this can be probably attributed to the variable cross-sectional area of the AM.

Next, let us compare the transmural distribution of the stresses at the AM locations (m) and (M) with the stresses predicted for the circular arterial segments $\Omega_{1 \lambda}^{\mathrm{C}(\mathrm{m})}$ and $\Omega_{1 \lambda}^{\mathrm{C}(\mathrm{M})}$. The stress distribution in Fig. $11 \mathrm{~b}$ that corresponds to the circular arterial segment is shown in blue. At position (m), the stresses predicted for the AM are somewhat higher in comparison to the stresses of $\Omega_{1 \lambda}^{\mathrm{C}(\mathrm{m})}$, whereas the opposite is true for the position (M). Such an error between the stresses predicted for the AM and for the circular arterial segments is a result of the AM variable cross-sectional area. The behaviour of the individual AM cross sections or segments is limited by the behaviour of the whole AM. Therefore, the individual AM segments cannot extend freely as is the case of the circular arterial segment. The error between the maximal circumferential stresses predicted for the circular arterial segments and those predicted for the $\mathrm{AM}$ is around $30 \%$.

\subsubsection{AM Loaded State}

The predicted AM loaded state $\left(\Omega_{2}^{\mathrm{AM}}\right)$ is presented in Fig. 12a. The stresses, as seen in the figure, are somewhat higher in the areas where the arterial crosssectional area is larger. The maximal circumferential stress $\sigma_{\phi \phi}$ is $73 \mathrm{kPa}$, which appears on the outer surface of the AM wall at the AM location $\left(M_{y}\right)$ (see the graphs in Fig. 12b). The range of stresses $\sigma_{\phi \phi}$, as well as their transmural distribution (Fig. 12b), agree with the corresponding data from literature [24] and [28].

Next, let us see the stresses that would be predicted for the AM sections (m) and (M) if they were treated as straight circular segments. The loaded state of the circular arterial segments (m) and (M), presented as $\Omega_{2}^{\mathrm{C}(\mathrm{m})}$ and $\Omega_{2}^{\mathrm{C}(\mathrm{M})}$, respectively, are shown in blue in Fig. 12b. The loaded states of both circular arterial segments (m) and (M) are computed as presented in Section 2.1.

Similar to what has been observed in the previous subsection, the stresses predicted for the AM at the position (m) are somewhat higher in comparison to $\Omega_{2}^{\mathrm{C}(\mathrm{m})}$, whereas the opposite is true for the position (M). The reason for this lies in the AM variable cross section which prevents the individual cross sections of the AM to extend freely. The error between the stresses predicted for the circular arterial segments and for those of the AM can be as high as $15 \%$. Given the size of the error, the suitability of using the circular arterial segments in order to determine the stress states of arteries, as is common in literature, is questionable.

\subsubsection{Predictions of the AM Zero-Stress State}

A way to partially verify the adequacy of the presented method is to simulate the opening angle experiment. If the stresses predicted for the arterial opened-up configuration are almost null, the method can be considered adequate. The same approach, for instance, can be seen in [2].

The zero-stress state of the $\mathrm{AM}\left(\Omega_{0}^{\mathrm{AM}}\right)$ is predicted based on the AM stretched state $\left(\Omega_{1 \lambda}^{\mathrm{AM}}\right)$ in two steps. First, the axial constraint that is applied to the $\Omega_{1 \lambda}^{\mathrm{AM}}$ state is released. The AM thereby contracts axially and occupies its unloaded state $\Omega_{1}^{\mathrm{AM}}$. In addition, the AM zero-stress state is obtained with a longitudinal cut which is performed in a numerical analysis by releasing the constraint on one of the AM symmetry surfaces (located on the $x-z$ plane, see Fig. 13a). The predicted equilibrium state of the AM opened-up configuration, obtained with a single longitudinal cut is designated as $\Omega_{0}^{\mathrm{AM} \# 1}$ and 
(a)

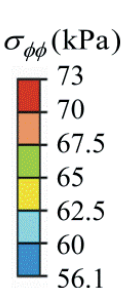

(b)

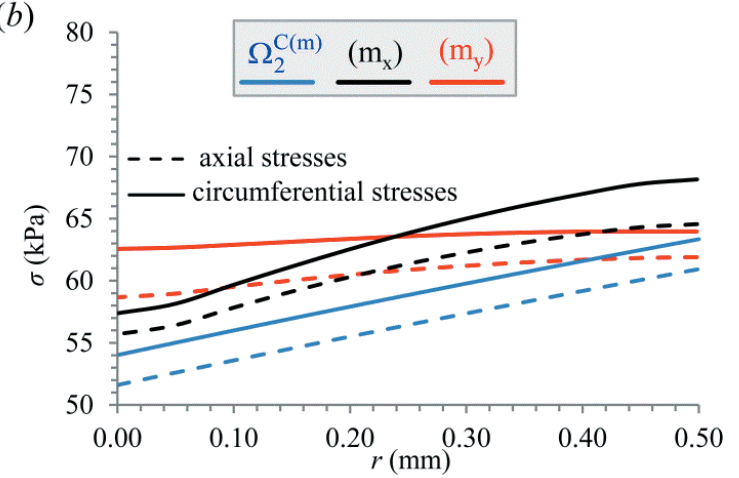

$\Omega_{2}^{\mathrm{AM}}(3 / 4$ view $)$
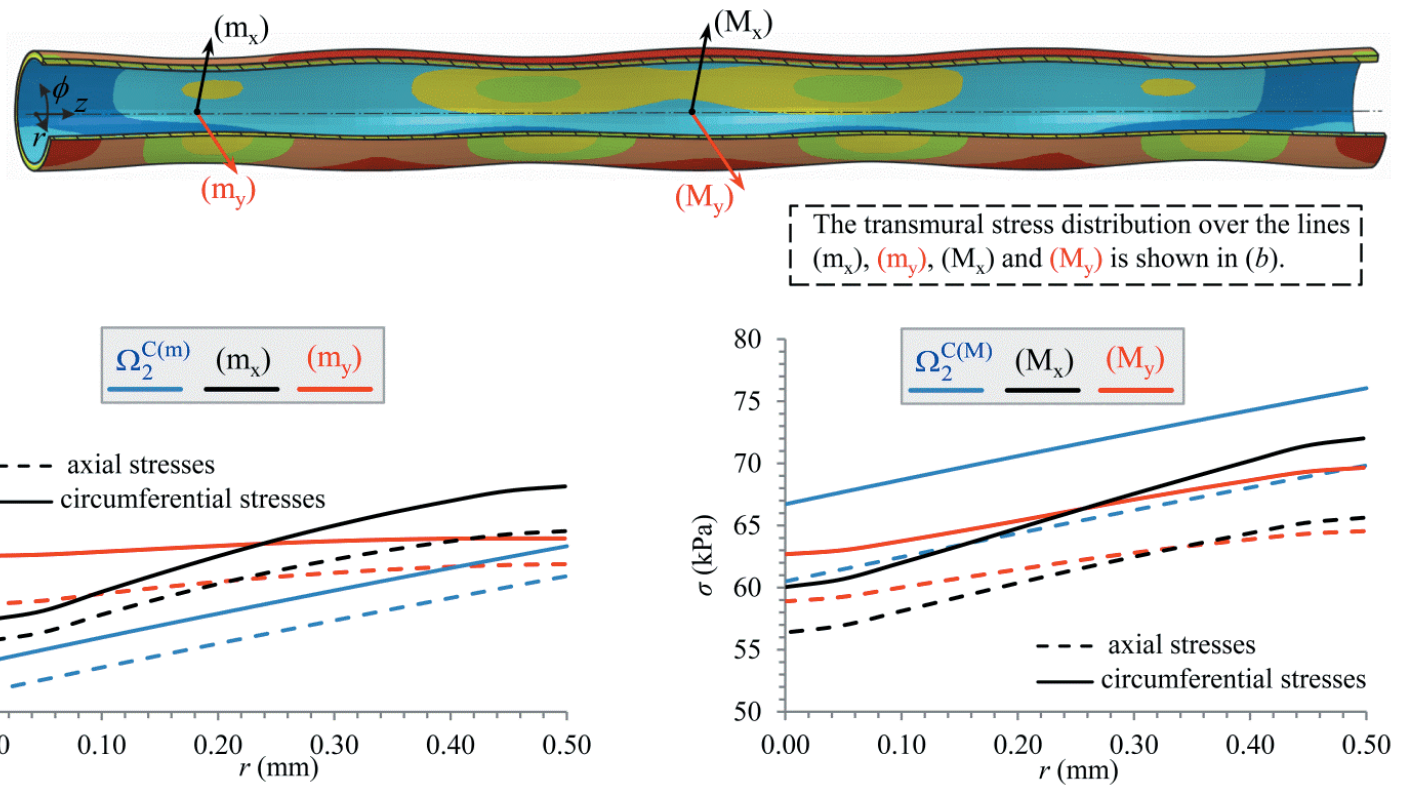

Fig. 12. The predicted AM stretched state $\left(\Omega_{1 \lambda}^{\mathrm{AM}}\right)$ : a) $3 \mathrm{D}$ view and b) the transmural distribution of the circumferential and axial stresses at positions $\left(\mathrm{m}_{\mathrm{x}}\right),\left(\mathrm{m}_{\mathrm{y}}\right),\left(\mathrm{M}_{\mathrm{x}}\right)$, and $\left(\mathrm{M}_{\mathrm{y}}\right)$ : their location is presented in Fig. 12a

(a)

$\Omega_{0}^{\mathrm{AM} \# 1}$

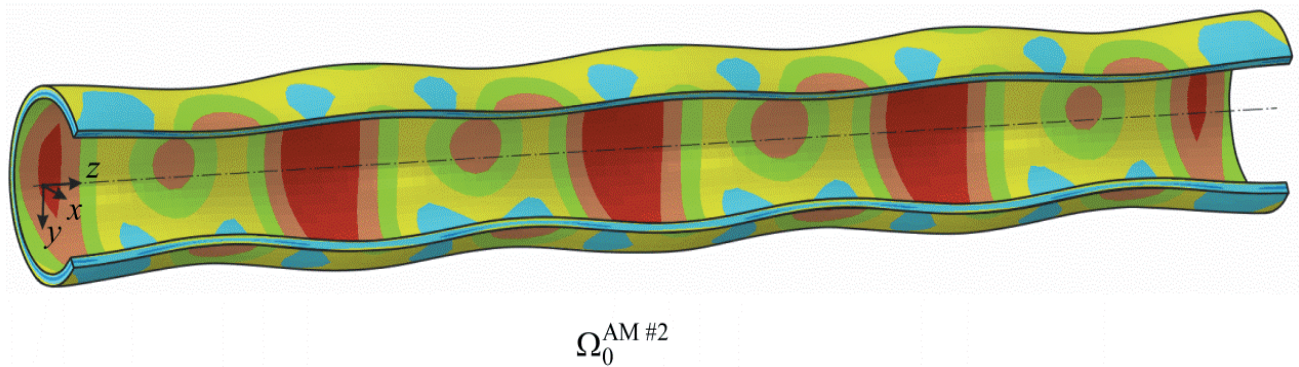

(b)

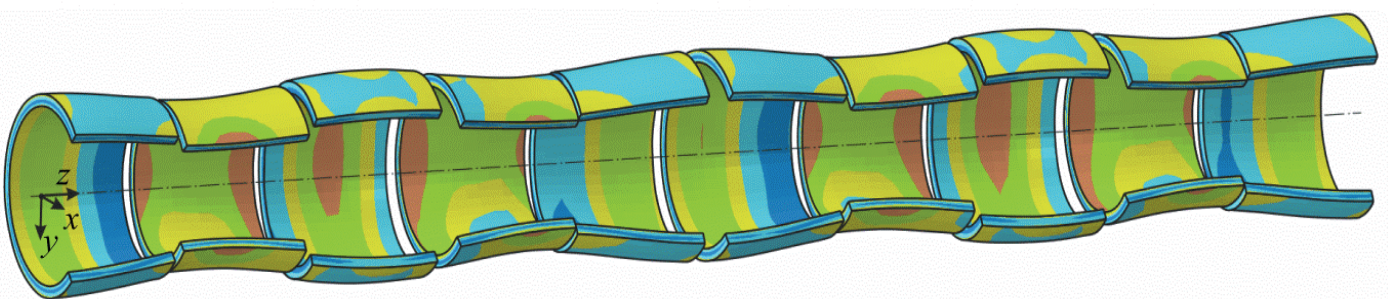

Fig. 13. Predictions of the AM zero-stress state: a) zero-stress state $\Omega_{0}^{\mathrm{AM} \# 1}$, obtained by longitudinal cutting of the AM unloaded state, and b) zero-stress state $\Omega_{0}^{\mathrm{AM} \# 2}$, obtained by transversely cutting the predicted $A M \Omega_{0}^{\mathrm{AM} \# 1}$ state into smaller segments

is presented in Fig. 13a. The maximal von Mises equivalent stresses in $\Omega_{0}^{\mathrm{AM} \# 1}$ are around $1.2 \mathrm{kPa}$ (Fig. 13a). These stresses occur locally in the areas where the AM cross-sectional area is larger. As seen in Fig. 13a, the predicted AM $\Omega_{0}^{\mathrm{AM} \# 1}$ state is not stressfree. The reason for this is that the AM variable cross- sectional area limits the AM's ability to extend freely, and consequently, it prevents the AM from occupying its zero-stress configuration. In order to obtain a better estimation of the AM zero-stress state, the predicted AM $\Omega_{0}^{\mathrm{AM} \# 1}$ state would have to be cut into smaller segments. This procedure is presented next. 
The predicted AM zero-stress state $\Omega_{0}^{\mathrm{AM} \# 2}$, which is obtained by cutting the $\Omega_{0}^{\mathrm{AM} \# 1}$ state into 10 separate sections, is presented in Fig. 13b. As seen in the figure, the maximal stresses obtained for the individually cut segments $\left(\Omega_{0}^{\mathrm{AM} \# 2}\right.$ state) are less than $1 \mathrm{kPa}$. These stresses occur in the second, third, and fourth segments (from both ends) whereas the maximal stresses range up to $0.8 \mathrm{kPa}$ in the remaining ones (the first and the fifth segment from both ends). For these segments, the stresses are lower by approximately $30 \%$ compared to the $\mathrm{AM} \Omega_{0}^{\mathrm{AM} \# 1}$ state. Higher stresses that appear in the second, third, and fourth segments are the result of the shape of the segments, which is, if compared to the first segment, somewhat curved in the $z$ direction. Based on the results presented, however, and given that the stresses of the AM $\Omega_{0}^{\mathrm{AM} \# 2}$ state are relatively low, it is possible to conclude that the use of the thermomechanical analogy in order to enforce the residual (bending and stretching) stresses into arterial model is justified.

\section{CONCLUSION}

In this paper, a method is presented which determines the arterial mechanical response without knowing the arterial opened-up zero-stress configuration. The arterial configuration on which the method is based is the arterial loaded configuration. We approach the problem of predicting the arterial residual (bending and stretching) stresses by considering a thermomechanical analogy. These stresses are determined by thermally loading a clamped, closed tube (called a fictitious model). The initial geometry of the fictitious model is constructed based on the luminal surface of the observed artery. The consequence of the applied thermal loading is that the fictitious model shrinks and the thermal (residual) stresses occur. In this paper, we have demonstrated that the loaded state of the fictitious model (i.e., its geometry and its stress state) is equivalent to the stretched state of the arterial model (see Section 4.1). However, in order for the presented analogical procedure to be adequate, the parameters of the fictitious model need to be properly defined.

The adequacy of the method has been verified by computing the zero-stress state of the arterial model. As presented in Fig. 13, the equilibrium configurations of the segments that are obtained following a longitudinal and transversal cut of the arterial model have the form of an opened-up tube. Furthermore, the residual stresses remaining in the segments are fairly mild compared to the stresses of the arterial stretched state. The results obtained show that the residual stresses predicted for the arterial stretched state with the thermomechanical analogy exhibit what is known as the bending distribution. This has proved the method to be adequate.

An interesting aspect that aroused in the analysis of the results is suitability of using the straight circular arterial segment for the characterisation of the arteries. This is a common approach in the literature [19], [28] and [29]. The segment is considered straight and stand-alone, meaning that the effect of the remaining artery in the segment's axial direction is not taken into account. Based on our results (see Section 4.2.2), the error between the stresses that are predicted for a straight circular arterial segment, and the stresses predicted for the arterial model, can be as high as $15 \%$. Additionally, the gradient of transmural distribution of the stresses for both cases differs (see Fig. 12). Nevertheless, it has to be said that a realistic artery does not exhibit such a variable cross-sectional area as was considered in this article. In a realistic artery, in fact, the influence of neglecting the effect of the remaining artery is smaller when observing one straight circular segment as a separate unit.

The results of the methodology presented in the paper show a distinction between the mechanical state predicted for a circular segment that is observed as a separate unit and the mechanical state that is predicted when the arterial variable cross-sectional area is taken into account. This topic therefore warrants further research, and presents one of the goals of our further work. Moreover, some of the arterial properties that have been neglected in this work, such as their multilayered structure or associated longitudinal residual stress, are planned to be implemented further in the methodology.

As for the clinical applicability of the presented methodology, the methodology could - supported by the medical imaging techniques - serve as a useful tool for predicting the patient-specific arterial mechanical response.

\section{REFERENCES}

[1] Holzapfel, G.A., Sommer, G., Auer, M., Regitnig, P., Ogden, R.W. (2007). Layer-Specific 3D residual deformations of human aortas with non-atherosclerotic intima thickening. Annals of Biomedical Engineering, vol. 35, no. 4, p. 530-545, DOI:10.1007/s10439-006-9252-z.

[2] Alastrué, V., Pena, E., Martinez, M.A., Doblare, M. (2007). Assessing the use of the "opening angle method" to enforce residual stresses in patient-specific arteries. Annals of Biomedical Engineering, vol. 35, no. 10, p. 1821-1837, DOl:10.1007/s10439-007-9352-4. 
[3] Vossoughi, J. (1992). Longitudinal residual strains in arteries. Proceedings of the 11th Southern Biomedical Engineering Conference, Memphis.

[4] Wang, R., Gleason, R.L. (2010). A mechanical analysis of conduit arteries accounting for longitudinal residual strains. Annals of Biomedical Engineering, vol. 38, no. 4, p. 1377 1387, DOI:10.1007/s10439-010-9916-6.

[5] Fung, Y.C., Liu, S.Q. (1989). Change of residual strains in arteries due to hypertrophy caused by aortic constriction. Circulation Research, vol. 65, no. 5, p. 1340-1349, D0I:10.1161/01.RES.65.5.1340.

[6] Humphrey, J.D. (2002). Cardiovascular Solid Mechanics: Cells, Tissues, and Organs. Springer-Verlag, New York, DOI:10.1007/978-0-387-21576-1.

[7] Bazilevs, Y., Hsu, M.C., Zhang, Y., Wang, W., Kvamsdal, T., Hentschel, S. Isaksen, J.G. (2010). Computational vascular fluid-structure interaction: methodology and application to cerebral aneurysms. Biomechanics and Modeling in Mechanobiology, vol. 9, no. 4, p. 481-498, Dol:10.1007/ s10237-010-0189-7.

[8] Fung, Y.C. (1991). What are the residual stresses doing in our blood vessels? Annals of Biomedical Engineering, vol. 19, p. 237-249, DOI:10.1007/BF02584301.

[9] Chuong, C.J., Fung, Y.C. (1983). Three-dimensional stress distribution in arteries. Journal of Biomechanical Engineering, vol. 105, no. 3, p. 268-274, Dol:10.1115/1.3138417.

[10] Chuong, C.J., Fung, Y.C. (1986). On residual stresses in arteries. Journal of Biomechanical Engineering, vol. 108, no. 2, p. 189-192, Dol:10.1115/1.3138600.

[11] Takamizawa, K., Hayashi, K. (1987). Strain energy density function and uniform strain hypothesis for arterial mechanics. Journal of Biomechanics, vol. 20, no. 1, p. 7-17, Dol:10.1016/0021-9290(87)90262-4.

[12] Bustamante, R., Holzapfel, G.A. (2010). Methods to compute $3 D$ residual stress distributions in hyperelastic tubes with application to arterial walls. International Journal of Engineering Science, vol. 48, p. 1066-1082, D0l:10.1016/j. ijengsci.2010.06.005.

[13] Greenwald, M., Moore, J., Rachev, A., Kang, T., Meister, J.J. (1997). Experimental investigation of the distribution of residual strains in the artery wall. Journal of Biomechanical Engineering, vol. 119, no. 4, p. 438-444, DOl:10.1115/1.2798291.

[14] Gee, M.W., Förster, Ch., Wall, W.A. (2010). A computational strategy for prestressing patient-specific biomechanical problems under finite deformation. International Journal for Numerical Methods in Engineering, vol. 26, no. 1, p. 52-72, DOI:10.1002/cnm.1236.

[15] Govindjee, S., Mihalic, P. (1996). Computational methods for inverse finite elastostatics. Computer Methods in Applied Mechanics and Engineering, vol. 136, no. 1-2, p. 47-57, DOI:10.1016/0045-7825(96)01045-6.

[16] Lu, J., Zhou, X., Raghavan, M.L. (2008). Inverse method of stress analysis for cerebral aneurysms. Biomechanics and Modeling in Mechanobiology, vol. 7, no. 6, p. 477-486, Dol:10.1007/s10237-007-0110-1.

[17] Delfino, A., Stergiopulos, N., Moore, J.E.Jr., Meister, J.J. (1997). Residual strain effects on the stress field in a thick wall finite element model of the human carotid bifurcation. Journal of Biomechanics, vol. 30, no. 8, p. 777-786, Dol:10.1016/ S0021-9290(97)00025-0.

[18] Raghavan, M.L., Trivedi, S., Nagaraj, A., McPherson, D.D., Chandran, K.B. (2004). Three-dimensional finite element analysis of residual stress in arteries. Annals of Biomedical Engineering, vol. 32, no. 2, p. 257-263.

[19] Holzapfel, G.A., Ogden, R.W. (2010). Modelling the layerspecific three-dimensional residual stresses in arteries, with an application to the human aorta. Journal of the Royal Society Interface, vol. 7, no. 46, p. 787-99, D0l:10.1098/ rsif.2009.0357.

[20] Schulze-Bauer, C.A.J., Holzapfel, G.A. (2003). Determination of constitutive equations for human arteries from clinical data. Journal of Biomechanics, vol. 36, no. 2, p. 165-169, DOl:10.1016/S0021-9290(02)00367-6.

[21] Masson, I., Boutouyrie, P., Laurent, S., Humphrey, J.D., Zidi, M. (2008). Characterization of arterial wall mechanical behavior and stresses from human clinical data. Journal of Biomechanics, vol. 41, no. 12, p. 2618-2627, D0l:10.1016/j. jbiomech.2008.06.022.

[22] Stålhand, J., Klarbing, A., Karlsson, M. (2004). Towards in vivo aorta material identification and stress estimation. Biomechanics and Modeling in Mechanobiology, vol. 2, no. 3, p. 169-186, D0I:10.1007/s10237-003-0038-z.

[23] Stålhand, J. (2009). Determination of human arterial wall parameters from clinical data. Biomechanics and Modeling in Mechanobiology, vol. 8, no. 2, p. 141-148, Dol: 10.1007/ s10237-008-0124-3.

[24] Cardamone, L., Valentin, A., Eberth, J.F., Humphrey, J.D. (2009). Origin of axial prestretch and residual stress in arteries. Biomechanics and Modeling in Mechanobiology, vol. 8, no. 6, p. 431-446, Dol:10.1007/s10237-008-0146-x.

[25] Abaqus 6.7.1 (2008). Users Manual. Simulia, Providence.

[26] Birger, B.I. (1972). Temperature stresses in an anisotropic cylinder, NASA, Washington.

[27] Fonck, E., Prod'hom, G., Ray, S., Augsburger, L., Rüfenacht, D.A., Stergiopulos, N. (2007). Effect of elastin degradation on carotid wall mechanics as assessed by a constituent-based biomechanical model. American Journal of Physiology - Heart and Circulatory Physiology, vol. 292, no. 6, p. H2754-H2763, DOl:10.1152/ajpheart.01108.2006.

[28] Stålhand, J., Klarbing, A. (2005). Aorta in vivo parameter identification using an axial force constraint. Biomechanics and Modeling in Mechanobiology, vol. 3, no. 4, p. 191-199.

[29] Masson, I., Beaussier, H., Boutouyrie, P., Laurent, S., Humphrey, J.D., Zidi, M. (2011). Carotid artery mechanical properties and stresses quantified using in vivo data from normotensive and hypertensive humans. Biomechanics and Modeling in Mechanobiology, vol. 10, no. 6, p. 867-882, DOI:10.1007/s10237-010-0279-6. 